\title{
Future Use of Large Eddy Simulation in Aeroengines
}

\author{
James C. Tyacke* \\ Whittle Laboratory \\ Department of Engineering \\ University of Cambridge \\ Cambridge \\ United Kingdom \\ Email: jct53@cam.ac.uk \\ Paul G. Tucker \\ Whittle Laboratory \\ Department of Engineering \\ University of Cambridge \\ Cambridge \\ United Kingdom
}

\begin{abstract}
Computational fluid dynamics (CFD) has become a critical tool in the design of aeroengines. Increasing demand for higher efficiency, performance and reduced emissions of noise and pollutants has focused attention on secondary flows, small scale internal flows and flow interactions. In conjunction with low order correlations and experimental data, RANS (Reynolds-Averaged Navier-Stokes) modelling has been used effectively for some time, particularly at high Reynolds numbers and at design conditions. However, the range of flows throughout an engine is vast, with most, in reality being inherently unsteady. There are many cases where RANS can perform poorly, particularly in zones characterised by strong streamline curvature, separation, transition, relaminarisation and heat transfer. The reliable use of RANS has also been limited by its strong dependence on turbulence model choice and
\end{abstract}

${ }^{*}$ Address all correspondence to this author. 
related ad-hoc corrections. For complex flows, Large-Eddy Simulation (LES) methods provide reliable solutions, largely independent of turbulence model choice and at a relatively low cost for particular flows. LES can now be used to provide in depth knowledge of flow physics, for example in areas such as transition and real wall roughness effects. This can be used to inform RANS and lower order modelling. For some flows, LES can now even be used for design. Existing literature is used to show the potential of LES for a range of flows in different zones of the engine. Based on flow taxonomy, best practices including RANS/LES zonalisation, meshing requirements and turbulent inflow conditions are introduced, leading to the proposal of a tentative expert system for industrial use. In this way, LES becomes a well controlled tool, suitable for design use and reduces the burden on the end user. The problem sizes tackled however have lagged behind potential computing power, hence future LES use at scale requires substantial progress in several key areas. Current and future solver technologies are thus examined and the potential current and future use of LES is considered.

\author{
Nomenclature \\ Re Reynolds number \\ $U \quad$ Velocity, $\mathrm{m} / \mathrm{s}$ \\ $\Delta x, y, z^{+}$Grid spacings in wall units \\ $\mathbf{x} \quad$ Coordinate direction \\ $\omega \quad$ Vorticity, $1 / \mathrm{s}$ \\ d Wall distance \\ $k \quad$ Turbulent kinetic energy \\ $i \quad$ Variable number \\ $\varepsilon \quad$ Turbulence dissipation rate
}

\title{
1 Introduction
}

The vast range of physical and geometrical effects that need to be taken into account, make turbomachinery one of the most challenging application areas for CFD. Sources of unsteady flow affect the efficiency and lifetime costs of the engine. Hence, unsteady numerical methods are critical in some flow regions to obtain satisfactory data. The numerous rows of rotors and stators generate boundary layer and secondary flows, encounter wakes, tip, seal and cavity leakage flows. Near the combustor, large vortical structures undergo rapid flow distortion and large temperature differences cause hot streak migration.

Large scale test facilities are disadvantaged by high preparation and operational costs [1]. A significant amount of time and money is also spent on the design, manufacture and maintenance of such facilities. Hence, numerical methods have long been used to provide a more economical alternative.

Secondary flows can have a significant effect on machine performance, for example, effects of tip leakage on stall [2]. Tyacke 


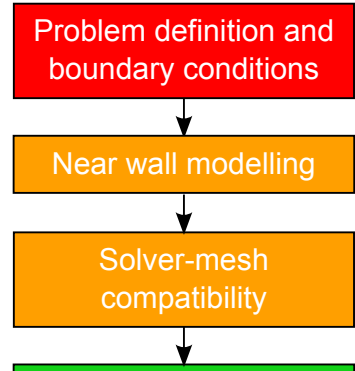

SGS modelling

Fig. 1: LES hierarchy indicating importance of each element.

Unsteady effects are often crucial, Unsteady-RANS (URANS) being limited by even advanced RANS turbulence models due to their dominating effect on the flow. This is especially apparent for heat transfer applications where errors of over 50\% can be encountered [3]. The calibration of (U)RANS to particular flows may make predictions inaccurate and unreliable outside of the calibrated region. Even so, their reliability and physical basis is often tenuous [4]. Notably, the existence or lack of a spectral gap in different engine zones impacts the theoretical basis of such closures.

Different modelling scenarios can lead to confusion over the use of relatively simple models as [5] versus more advanced turbulence closures [6,7], often involving additional corrections for physical effects or numerical anomalies [8,9]. Many RANS models and modifications are applied at idealised conditions to address particular deficiencies. Unfortunately, the very nature of aero-engines is that they involve complex flows of varying length and time scales, with numerous unsteady interactions.

Specific areas of difficulty include flow anisotropy, heat transfer, transition (including bypass, separation, wake and shock-induced) and re-laminarisation and regions of strong mixing such as internal cooling passages and surface and trailing edge cut-back film cooling. This advocates the use of eddy-resolving methods such as LES, hybrid RANS-(N)LES and even direct numerical simulation (DNS). Even though the cost compared to RANS simulation is at least $100 \times$ greater, the relatively low impact of LES turbulence closures on many complex flows and the data quality and detail provided are clearly advantageous.

Although Dettached Eddy Simulation (DES) [10] can alleviate substantial grid demand, it is prone to grid-induced separation and other difficulties [11]. For high $R e$ attached boundary layer flows, near wall effects are generally modelled well using RANS, hence a RANS layer used in the inner layer $\left(y^{+}<100\right)$ is beneficial in reducing grid requirements. This method also avoids grid dependence and has been used with success $[12,13]$ - although care can be required.

Other flow types such as free shear flows are ideal for LES, where scales are often similar to geometrical features or are large wake-type structures. Grid resolution is moderate for these flows and only weakly dependent on $R e$.

Figure 1 shows an LES hierarchy, with critical problem definition and boundary conditions indicated in red, important mesh-solver compatibility and near wall treatment in amber and SGS modelling regarded as low importance displayed in green. This is in contrast to RANS, where the turbulence model dominates the solution.

The Reynolds numbers of the order of $10^{5}$ and the availability of high performance computing (HPC) facilities has made LES of many turbomachinery flows feasible $[14,15]$. Chapman [16] estimated that when computing power reaches Tyacke 


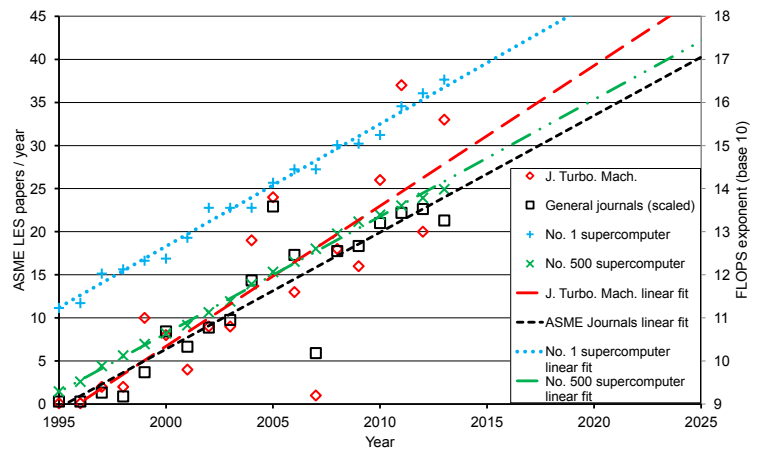

Fig. 2: Number of LES publications in turbomachinery compared to other disciplines and available computational power.

$10^{14}$ FLOPS, LES would begin to compliment and perhaps replace some rig-testing. Figure 2 shows the recent trends in the publications of LES use in turbomachinery (red long dash) contrasted with multi-disciplinary ASME journal publications (black short dash). The number of general ASME LES papers (scaled to the percentage of J. Turbo. Mach. articles) and computational performance are plotted along a time scale. The straight lines are linear curve fits. The number of journal papers published each year is correlated to the available computational power. In 2008, the world's number 1 supercomputer (blue dotted line) reached $10^{15}$ FLOPS. More significant is that the $500^{t h}$ fastest supercomputer (green dash-dot-dot) is due to reach the same level of performance circa. 2016. Exascale ( $10^{18}$ FLOPS) computing will be reached by the number 1 supercomputer before 2020 and the trends show the $500^{\text {th }}$ fastest is likely only 10 years behind. From Fig. 2 it is clear that LES is rapidly becoming more useful and accessible, though the problem size being tackled is lagging behind the available computational resources.

For LES methods to be used in industry, the crucial problem definition and case setup must be controlled to ensure best practice and consistency between users. Qiu et al. [17] present an integrated design system for turbomachinery including 1D-2D-3D CFD, Finite Element Analysis (FEA) and Computer Aided Manufacture (CAM). The need for computational resource is reduced by the appropriate use of different fidelity levels. Rubio et al. [18] use an Artificial Neural Network (ANN) to design an expert system that accurately predicts the number of iterations and runtime required for a CFD solution. This is useful for indicating the required time and costs involved. Rubio et al. highlight that user experience generally plays a large role in choosing turbulence models and estimating computational effort. Using the system, computation times were predicted to within approximately $13 \%$ with $68 \%$ confidence $(1 \sigma)$ and $25 \%$ with $95 \%$ confidence $(2 \sigma)$. Knight and Petridis [19] develop a knowledge-based system to aid in solution control, monitoring cell aspect ratio, solution type and grid quality to increase accuracy and convergence. Andrews [20], investigates the use of a range of Artificial Intelligence/CFD systems. It is possible to obtain a high return on investment depending on the existence and exploitation of knowledge for different cases. Relatively little effort can leverage the knowledge based in different codes but bottlenecks, formalising CFD methods and efficient automation are identified as challenges. Today we expect to be able to tackle some of these challenges with the great advances in computational science, power and intelligence.

The current paper is organised following loosely the hierarchy above. Boundar conditions and problem definition will be discussed, then mesh-solver compatibility and SGS modelling will be addressed. Future solver technology and grid Tyacke 


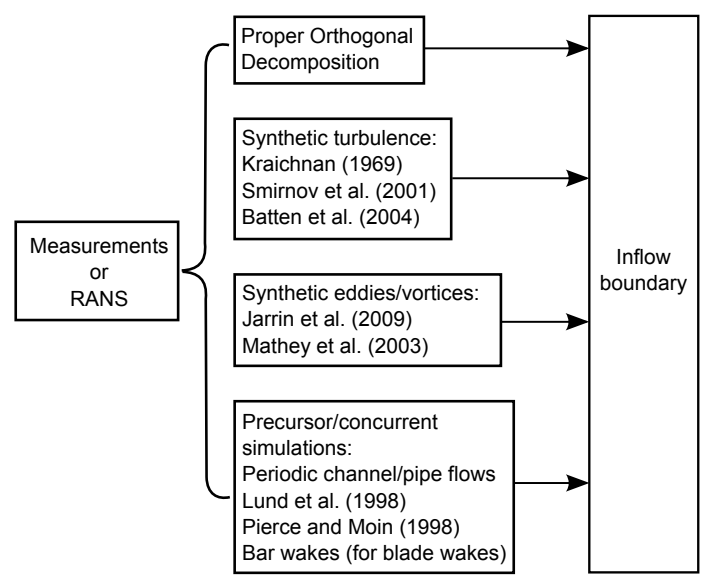

Fig. 3: Potential inflow options for idealised turbulence.

resolution requirements are examined and LES validation, flow taxonomy and best practices will be introduced. Finally, for future industrial use, a conceptual expert system and uses of LES will be discussed before drawing conclusions.

\section{Boundary Conditions and Problem Definition}

\subsection{Turbulent inflow}

LES requires boundary conditions that closely represent physical conditions. For example, the heat transfer for an HPT blade is sensitive to incoming turbulence scales, in contrast to RANS, which can wrongly be insensitive [21]. For flows lacking strong turbulence generating mechanisms i.e. highly inflectional velocity profiles or bluff geometrical features, sufficiently high quality inflow turbulence is important. Sagaut et al. [22] explore turbulence inflow techniques for a range of flows. For a wide cavity flow, it is noted that resolved turbulence in the inflow boundary layer is not so important. In a similar fashion to jets, there is a highly inflectional inflow velocity profile that allows the rapid development of resolved scales.

With complex inflow requirements, various methods are available to generate suitable turbulence fields. For reviews see [23-25]. Figure 3 shows key methods for obtaining inflow turbulence, some being intended for use within an industrial process. This represents only a small number of possibilities (the authors estimate there are at least 50 methods/variants), however most are idealised and do not represent the complex nature of flows throughout turbomachines. Some key elements relating to the relative performance of different inflow techniques are:

(a) How long a development length is needed to establish turbulence?

(b) How long (for recycling methods) is needed to overcome initial transients and thus establish a developed turbulent state?

(c) How practically usable is the method?

(d) How Reynolds number limited is the method?

(e) How well does the output connect with real engineering systems?

An obvious choice is to use existing experimental data, precursor simulations (or even concurrent simulations), that can Tyacke 


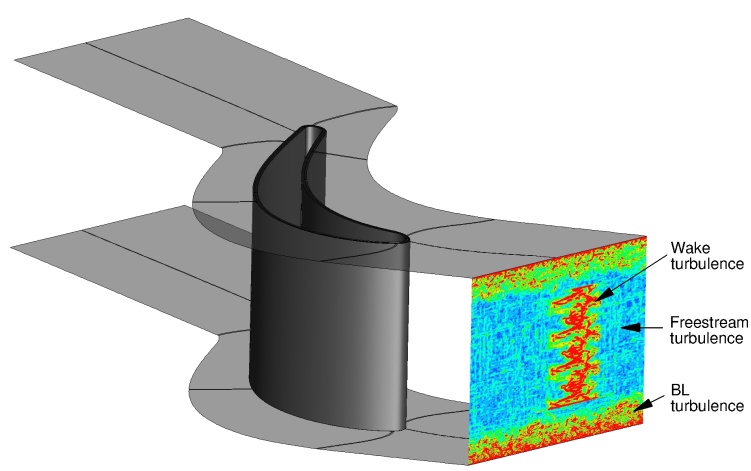

Fig. 4: Merging of wake, boundary layer and freestream turbulence for a LPT simulation (vorticity magnitude contours).

be stored in a database. However, although physically ideal, this seems impractical due to the diverse range of operating conditions throughout a turbomachine. Also, Reynolds number restrictions may also become a limiting factor in the range of operating points that can be achieved.

Another key method for generating turbulence inflow is through use of re-cycling procedures such as Lund's recycling [26], where iteratively, a flow plane is extracted between the inlet and outlet, scaled and imposed at the inlet until the required boundary layer conditions are met. Although the basic approach sounds in essence simple, there are potential implementation difficulties [23] and hence variants. A simpler alternative can involve simple pipe or channel flow LES/DNS with periodic flow boundary conditions giving rise to fully developed boundary layers. Schlüter et al. [27] apply a recycling approach to whole engine simulations. However, (for the compressor) RANS information is used to set/scale the mean velocity and turbulence levels in the recycling procedure. The latter just provides temporal information. For many practical flows, there can be classical boundary layer content and also external flow activity. This could include wakes from turbine blades along with inter-wake turbulence. When Lund's recycling is used, these coupled elements, would need to be blended and scaled in some plausible way. $\mathrm{Wu}[28]$ terminates his wake modelling at some multiple of the momentum thickness away from the boundary layer. For LPT simulations, Vadlamani [29] combines Lund's recycling with wakes (from Wu) and also isotropic box turbulence (for the inter-wake zone external to the boundary layer). Figure 4 shows the merging of these components at the simulation inflow plane. In the figure, the blending of these components is less gradual. This helps identify the three different inflow components. For hybrid RANS-LES, if unmodified, Lund's recycling would force resolved scales into the RANS zone, which is undesirable. The decoupled procedures discussed above fail to account for the boundary layer modification from the external flow. As noted by Mathey et al. [30], multiple flow boundaries will only exacerbate these problems. Hence, recycling procedures may only be practically useful for flows only containing boundary layers.

Synthetic turbulence can be generated based on RANS information or even measurements [31-33]. A key problem with such approaches is that there can be too rapid a decay of the imposed turbulence. This is evident in the LPT LES of O'Mahoney [34]. Keating et al. [24] show inflow scales should be of the order of the integral length and time-scale $\left(1-4 L_{0}\right)$ as the smaller scales react rapidly to produce the correct turbulence, also implying only a few modes may be needed. To Tyacke 


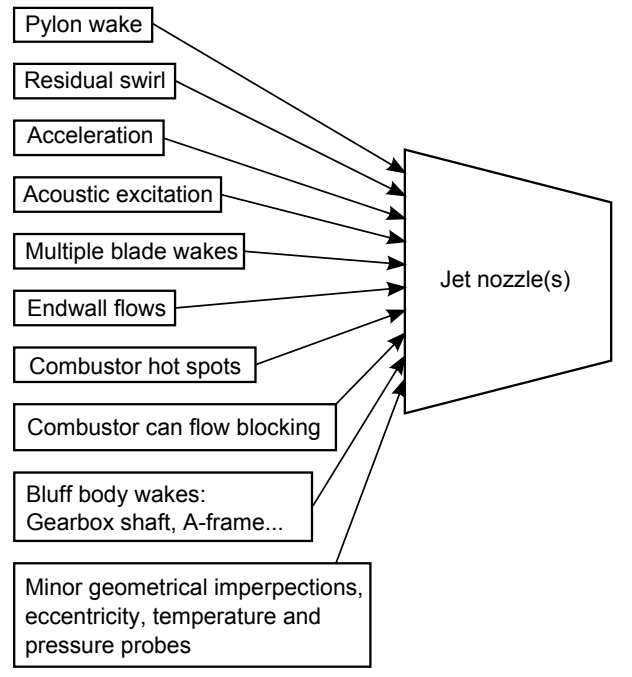

(a)

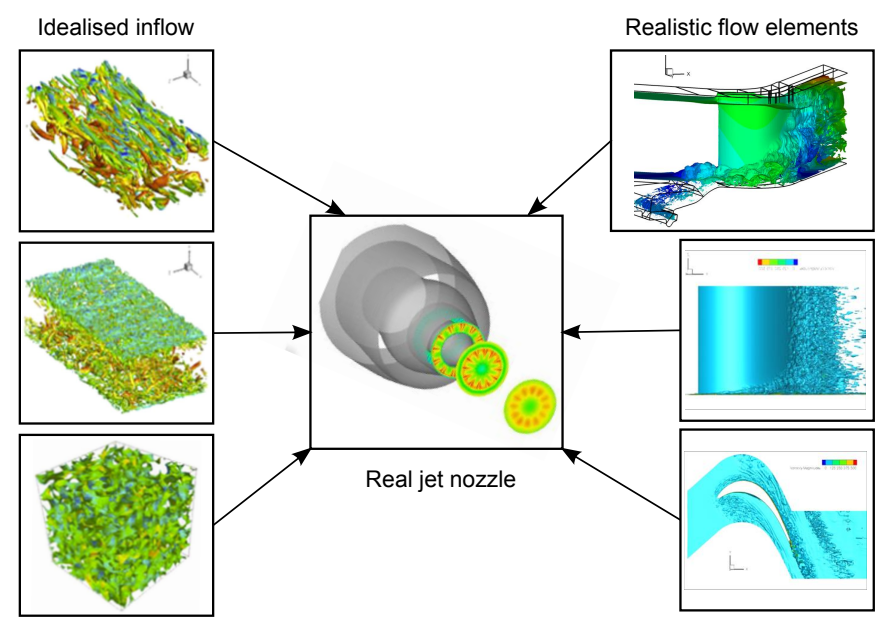

(b)

Fig. 5: Inputs to flow field in a real propulsive jet nozzle: (a) potential inputs for real jet nozzles, (b) idealised and realistic inputs.

overcome problems of rapid decay, downstream control zones, where forcing is imposed (see [24]), can be used.

With respect to cost and user ease, synthetic turbulence generally seems a preferable option. However, for simple boundary layers, synthetic turbulence will be less authentic than recycled. Therefore, a longer development zone will generally be needed. The digital filtering approach [35], synthetic eddy generation [36] and proper orthogonal decomposition based approaches (see $[37,38]$ ) are also relatively popular methods for generating turbulence inflow.

Element (e), in the list above i.e. how well the output from idealised turbulence generation techniques connects with that found in real engineering systems, is critical. Many of the more idealised inflow approaches, noted above, in their raw form, have low relevance to turbomachinery. For example, it might be tempting to consider applying Lund's recycling, to jet nozzle flows. For the replication of laboratory experiments this has some value. However, a real nozzle has various upstream influences. As shown in the LES work of Eastwood [39],the influence of upstream blade rows and the pylon can have a substantial influence on the jet development. Figure 5a further illustrates the numerous sources of inlet turbulence that could be considered for a jet nozzle. Similar diagrams could be produced for other engine zones. Figure $5 \mathrm{~b}$ contrasts idealised inflow turbulence on the left and more realistic geometries and turbulence generated upstream of a propulsive jet. There are clear differences in the type of inlet turbulence that is often generated a priori (left) and that which represents true flow conditions (right). Clearly, imposing rather idealised turbulence fields has limitations in such a large-scale, coupled, system as a gas turbine engine. Many rig tests also suffer from the same lack of proper inflow and outflow characterisation that LES does. For example, for jet rigs the state of boundary layers is frequently unknown. This, in turn, hampers the assessment of LES due to poor problem definition.

Approaches similar to that of Batten et al. [40], perhaps, seem most realistic in a practical engineering context. In this case, large-scale upstream (U)RANS simulations can be performed to generate synthetic turbulence inflow. Based on (U)RANS, turbulent flow can be generated with scales and structure resembling those required in an automated fashion. The latter aspect will be critical if coupling zones modelled with different fidelity methods. Another possibility is to use RANS 


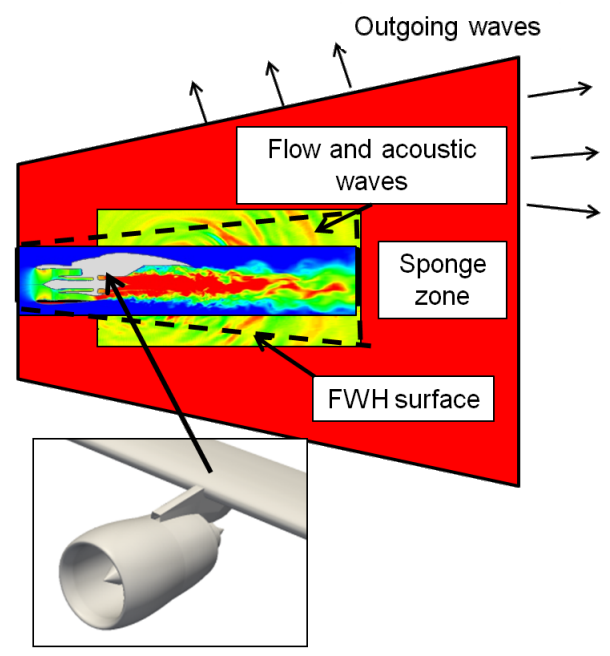

Fig. 6: Jet LES with sponge zones indicated in red.

in conjunction with a recycling or forcing procedure. The RANS provides target statistics and recycling or forcing is used to generate a flow requiring little downstream development [41,42], (though these examples require a virtual recycling zone).

\subsection{Outflow}

For the application of LES to real turbomachinery systems, the vision for the development of both inflow and outflow boundary conditions perhaps needs extending. Like inflow, outflow needs to consider the complex, multi-component, coupled nature of the turbulence fields produced. As an example, wake shedding from HPT blades introduces upstream and downstream vortical, entropy and pressure waves which interact with other flow regions [21]. This potentially excludes the common use of grid coarsening, flow smoothing and domain extension to prevent wave reflections at boundaries [43-45]. To use smaller domains and avoid non-physical solutions, non-reflecting boundary conditions are required. One dimensional non-reflecting boundary conditions include those of Giles [46]. Pauley [47] presents a convective boundary condition that is easy to implement relying on the bulk mean velocity. The flow and acoustic fields are three-dimensional, hence the previous approaches require extending to more general cases. Specification of a target field is another option, with a transition zone as in Ashcroft and Zhang [48].

For jet flow and acoustic fields, additional dissipation can be introduced away from the active flow and acoustic source regions as a sponge zone. This is shown in Fig. 6 by the red region. Acoustic waves generated in the near field are recorded at the Ffowcs-Williams Hawkings [49] (FWH) surface before being dissipated in the sponge zone. Alternatively, a radiative boundary condition can be applied based on the asymptotic solutions at the farfield as by Tam [50]. This two-dimensional method can be extended to three-dimensions though large domains are usually required. Use of the perfectly matched layer approach of $\mathrm{Hu}$ [45] allows a smaller sponge layer near boundaries to be used as out going waves are absorbed. This also addresses limitations of essentially 1D characteristic based conditions, where waves are usually assumed normal to the boundary. At domain corners or for internal flows with multiple boundaries this is often not the case. Although still based on 1D approximations, the NSCBC method of Poinsot and Lele [51] may offer a compromise between performance 
and complexity. Further research is required to provide accurate non-reflecting boundary conditions for more complex geometries such as the turbine, where multiple waves meet multiple boundaries at varying angles.

\subsection{Coupling}

Substantially beyond the use of normal blade row sliding planes, two-way coupled systems are becoming increasingly important. Most experimental engine intake duct studies do not include upstream rotors or a downstream compressor. Also, the HPT affects all subsequent stages, the unsteady thermal loading potentially affecting fatigue life [52]. Hence the assumptions and modelling in different engine zones could have a strong overall impact on design and performance. Care is also needed when comparing with experimental data. In the work of $\mathrm{Li}$ [38] the compressor outlet guide vane and prediffuser zone of a gas turbine engine is considered. The latter connects to the combustion system. As shown by Li, for optimal diffuser design it is necessary to consider these two zones in combination. The performance of these two elements will, in turn have a strong impact on the combustor external aerodynamics. The wakes from the outlet guide vane (and even the rotors upstream of these) will influence the diffuser performance, which in its optimal state will be sensitive to the form of incoming boundary layers. Li performed LES applying both recycling and a recycling with body force based procedure related to that of Pierce and Moin [53]. Pierce and Moin used body forces to produce a swirl component. Along, similar lines, Eastwood [39] used body forces to represent blades upstream of a propulsive jet in hybrid RANS-LES work. Through calibration these provided realistic and economical wake turbulence.

For two-way blade row coupling using LES, sliding planes may be used to transfer unsteady data across the interface. As mentioned, to couple zones of RANS and LES, some degree of forcing may be required.

Engine zones can potentially be coupled using RANS and LES as [27,54,55]. In [27] both compressible, incompressible, structured and unstructured solvers are run simultaneously and interfaced using custom message passing interface (MPI) communicators. RANS and LES regions are linked using fluctuations from a database scaled to the upstream RANS zone for LES inlet and body forces at the LES zone outlet to meet the downstream steady RANS conditions. For LES embedded in a RANS zone, Patil [56] uses a synthetic eddy method to obtain inlet turbulence, having a significant beneficial impact on accuracy for a combustor. At the combustor-turbine interface, pressure-based and compressible solvers may require coupling, demonstrated by Medic et al. [57] who model a sector of a whole engine using different levels of fidelity. To ensure parallel scalability, different solvers will require close integration. Complex RANS simulations may also contain, localised embedded LES zones [58].

Other specific types of coupling include fluid-structure, acoustic interaction and conjugate heat transfer. Example physics coupling is shown in Fig. 7, where $T_{\text {solid }}^{*}$ and $T_{\text {fluid }}^{*}$ represents the thermal time constant of the solid and fluid. Conjugate heat transfer problems, such as those found in the HPT are often numerically stiff due to the disparity in air to metal thermal time scales [59]. Novel methods such as Fourier Spectral Modelling may be used to tackle these issues [52]. More generally, interfaces between modelling fidelity disparities should remain conservative, accurate and be non-aliasing. The management of uncertainties and sensitivities across different zones and disciplines will become increasingly important. To relieve the computational burden of many coupled simulations, LES could be useful in developing more accurate lower 


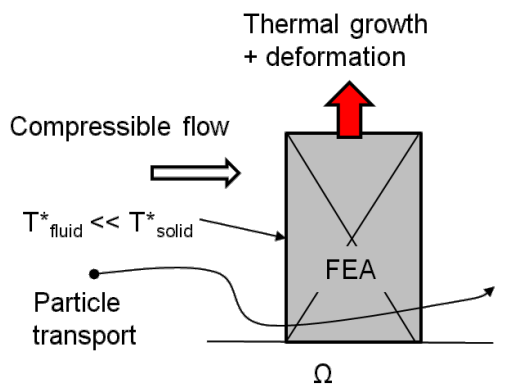

Fig. 7: Example of physics coupling for a turbine passage.

order methods.

\subsection{Specialised Turbomachinery Boundary Conditions}

For turbomachinery blade rows, full annulus simulations are impractical and smaller domains are sought. In contrast to URANS, LES is not periodic in time due to stochastic components. Blade count mismatches often prevent the use of pitchwise periodicity. In these cases phase lagged boundary conditions could be used. Here, the time history is stored and lags in time between blade rows. However, this can generate physically valid but implausible solutions where shedding appears to occur identically around the annulus, hence polluting an otherwise sound LES. Alternatively, time-inclining $[60,61]$ may be more appropriate with two-way axial coupling via a sliding plane (with compatible mesh densities and interpolation of variables). In this case, the time coordinate is transformed, being a linear function of circumferential location, blade pitch and rotor angular velocity. This approach also does not corrupt accuracy [62]. The different location of variables in time can be rectified during post-processing.

\section{Mesh, Solver and SGS Model Interactions}

The numerical aspects of current solvers can be characterised using a variety of canonical cases. For more dissipative solvers the use of NLES may be appropriate. Some discretisations give rise to formal implied models (the numerical equivalent to explicit SGS models) as is the case for MILES [63]. Non-dissipative schemes require some form of dissipation either numerically or using an explicit SGS model. Hence grid-solver compatibility is one of the primary concerns for LES.

Mesh generation for complex geometries is a key challenge in reducing the amount of user time and input for successful CFD and will likely remain so in the near future. For LES, aspects other than geometry representation are also important. Cell type and mesh topology are to an extent governed by the underlying numerical discretisation which should not introduce excessive artificial dissipation. Figure 8 shows the spatial decay amplitude error of a Tollmien-Schlichting (T-S) wave using different cell types. The large range of amplitudes and hence dissipation between cell-types compared to the analytical solution of the Orr-Sommerfeld equation [64] is clear. The temporal and spatial discretisation (for example flux evaluation) also plays a critical role in the numerical traits observed in a given solver. Figure 9 shows the variety and popularity (see [44]) of spatial and temporal discretisations available. The spatial schemes include Essentially Non-Oscillatory (ENO), Weighted Essentially Non-Oscillatory (WENO), Advection Upstream Splitting Method (AUSM), Monotone Upstream-centered Schemes 


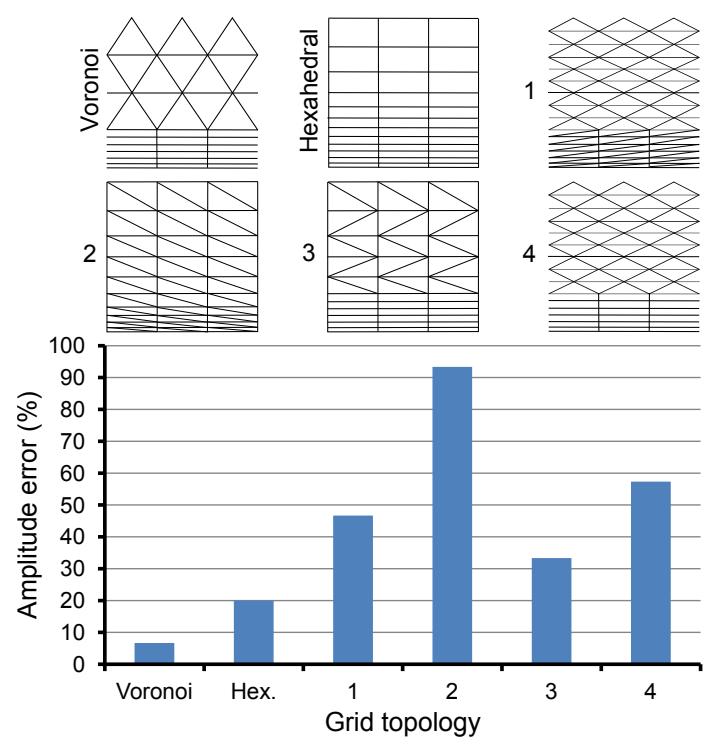

Fig. 8: Grid topology influence on T-S wave decay. \% Error in amplitude at circa. 50\% domain length.

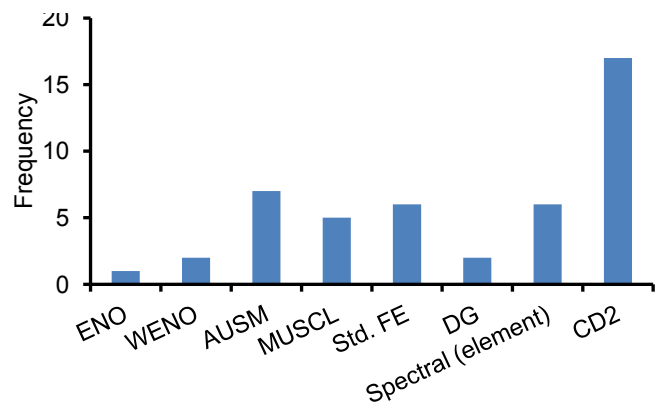

(a) Spatial scheme

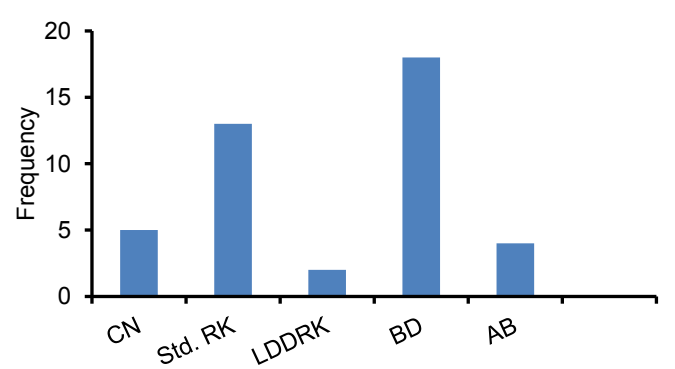

(b) Temporal scheme

Fig. 9: Use of numerical schemes in literature.

for Conservation Laws (MUSCL), Finite Element (FE), Discontinuous Galerkin (DG), Spectral/spectral element and Second order central difference (CD2). The temporal schemes (see Fig. 9b) being Crank Nicolson (CN), Standard Runge-Kutta (Std. RK), Low Dissipation and Dispersion Runge-Kutta (LDDRK), Backward Difference (BD) and Adams-Bashforth (AB). In practice, spatially, most solvers use second order central differencing with, for example, Roe-type smoothing [65] for high speed flows to maintain numerical stability. Runge-Kutta, implicit backward and the Crank Nicolson schemes are most common for time discretisation, i.e. although there are a wide range of more advanced schemes, it is the more basic that see practical use. As can be seen from Frame (b) this is the case for the temporal scheme also. This may be a legacy of RANS solvers that have been shifted to parallel architectures and has resulted in solvers that have traditionally been considered Tyacke 


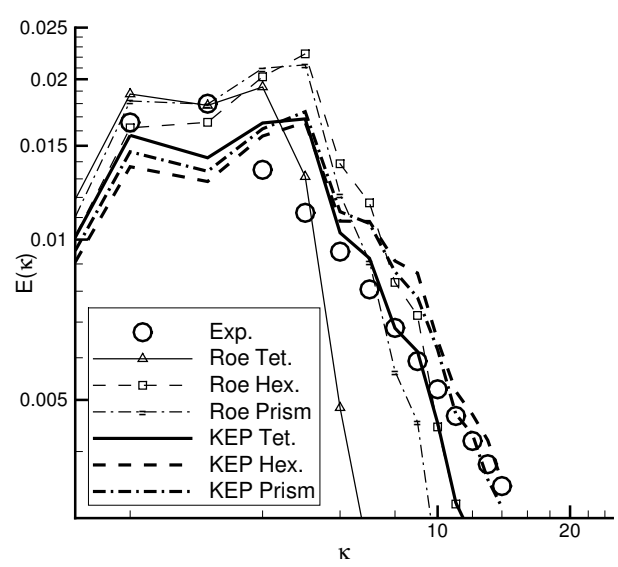

Fig. 10: Numerical influence on HDT.

fast. The effect of using lower order time discretisation than the spatial scheme can also be as significant as the order of the spatial scheme itself as shown by DeBonis and Scott [66]. Spatially, non-dissipative schemes such as the Kinetic Energy Preserving (KEP) scheme [67] have shown some advantage over typical second order Roe-based schemes as used above. Figure 10 shows the improved high frequency content captured using the KEP scheme for homogeneous decaying turbulence (HDT). The reduced dissipation allows a wide variety of cell types to be used effectively with only a small effect on the solution. In fact, the tetrahedral element result using the KEP scheme is superior to that of the hexahedral elements using the standard Roe-type scheme. In general, for flows involving shocks, some amount of local high-order upwinding $(O \geqslant 2)$ may be required to prevent non-physical oscillations. For LES, long time integrations suggest the need for fewer grid nodes, fewer time steps, lower numerical errors and improved scalability. Carefully selected and implemented higher order $(O>2)$ discretisations appear to be suitable methods to pursue. Methods also exist which may enable parallelism in time such as the parareal method [68], though this has seen little success for turbulent flows. However, the scalability of different spatial and temporal schemes may need to be re-assessed for emerging computational architectures to reach the best trade off between accuracy and performance.

It should be noted that the use of overset meshes in regions of interest to avoid high cell counts away from boundaries is required to meet the grid point requirements of Chapman [16,69]. Choi and Moin [70] also recently reached similar requirements for wall resolved LES ${ }^{1}$. High order discretisations can also be achieved relatively easily on structured overset meshes for Finite-volume and Finite-difference methods. When using Discontinuous Galerkin or Spectral element methods, high order discretisations can also be achieved with improved data locality in computer memory. Additional geometric detail such as cooling holes, trailing edge cutbacks, endwall profiling and multiple stages soon make the generation of quality meshes time consuming. Significant advances in automatic blocking topology for structured meshes is required for complex geometries. In lieu of this, immersed boundary methods (IBMs) [71,72] are an alternative approach to meshing complex problems, reducing pre-processing time by the inclusion of body forces to represent geometry. This seems especially attractive for wake-type flows (such as ribbed internal cooling ducts) that may be less sensitive to approximations in geometry representation. It will become increasingly important either to use meshing templates of common geometries and features,

\footnotetext{
${ }^{1}$ We note Chapman's outer layer estimates are optimistic. Tyacke
} 


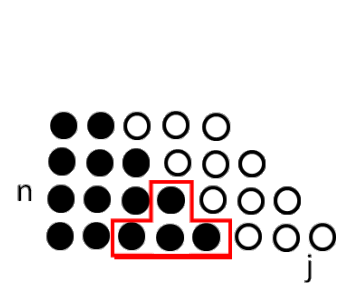

(a) Tiling

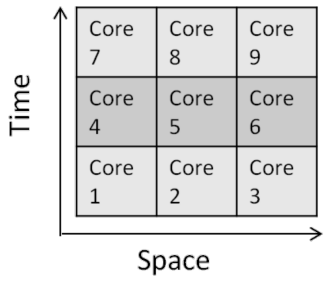

(b) Parareal

Fig. 11: (a) Tiling and (b) Parareal methods to avoid communication and increase prarallelism.

or a significant advance in the automatic generation of high quality meshes compatible with state-of-the-art LES solvers. To improve automation, more accurate, clean $\mathrm{CAD}$ geometry is also required in portable formats that can be used within many systems.

Where unstructured meshes are appropriate, meshes can rapidly be generated. However for LES, representation of the geometry only is inadequate. Generating the correct resolution mesh for different flow features a priori using unstructured methods is challenging. Improved quality criteria based on grid metrics and importantly, the flow will be required [73]. In addition, the use of solution adaptive meshes may be necessary to eliminate user oversights [74]. Advances in sensitivity analysis may also be required when assessing quality based on LES data [75]. If higher order methods are to be used, more accurate $\mathrm{CAD}$ and higher order grid elements may be required [76].

\section{Solver Technology and Emerging Architectures}

Chapman [16] assumed there would be significant progress in numerical algorithms, widening LES use. It seems progress has been slower than expected on this front. Löhner [77] and Giles [78] suggest 85-90\% of CPU cycles are wasted, mainly due to memory and network bandwidth constraints. In the past decade, CPUs have moved from single to multicore architectures and plugin cards are now readily available that provide the same performance per Watt of a national high performance computing (HPC) facility ten years ago [79]. Considering HPC, machines of many thousands or millions of cores are currently available and in future, architectures of 10 billion way parallelism or more can be expected [80]. The current wastage of performance, has a strong impact on the cost of hardware, power and time associated with simulations. Although some hardware improvements such as integrated photonics [81] will alleviate some bottlenecks and reduce power consumption, hardware alone will not be sufficient to close the void in attainable performance. There is hence a strong impetus to develop and efficiently implement algorithms that avoid communication, improve data locality to the compute units and reduce the amount of computation time.

Communication avoiding algorithms such as tiling [78] and potentially, time-parallel methods such as Parareal [68] may help in certain cases. In Fig. 11a, the data dependence enclosed in red requires no communication between the white and black nodes. Fig. 11b shows how a problem is split in space and time and computed on different computational cores (there is later a correction made to enforce causality in time). So far these methods have seen little use for general turbulent flows, but may be fruitful if data dependence is clearly identifiable for example. To relieve data bandwidth, implies performing 


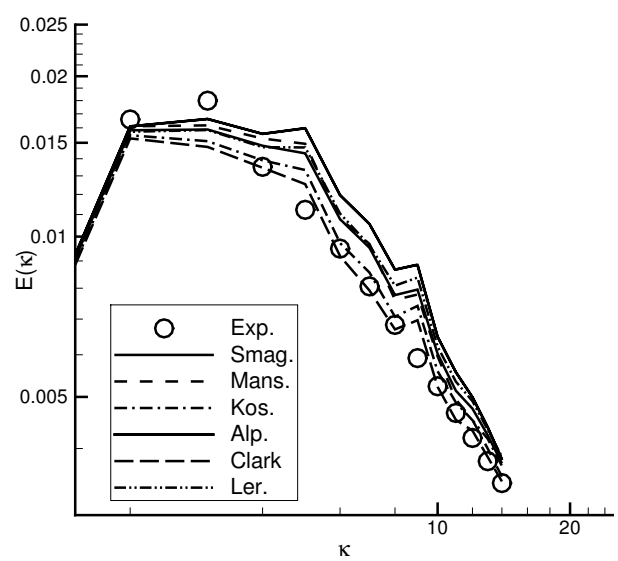

Fig. 12: HDT for various SGS models.

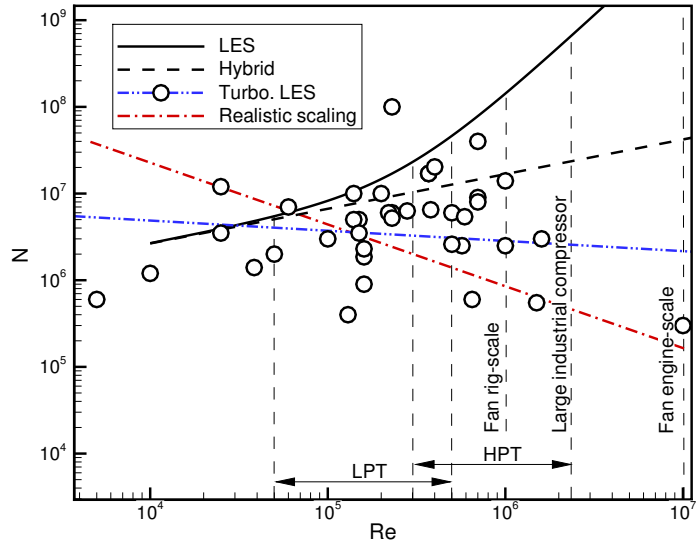

(a)

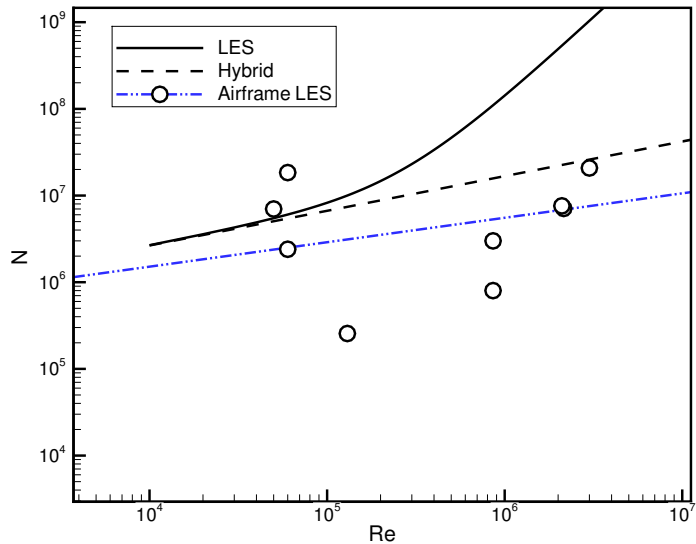

(b)

Fig. 13: Grid point requirements for LES and Hybrid RANS-(N)LES compared with typical grid densities used in existing literature for (a) Turbomachinery, (b) Airframe.

more local operations to make use of CPU cycles rather than avoiding computation by storing and transferring variables in memory.

\section{$5 \quad$ SGS Modelling}

As shown in Fig. 1 in the introduction, SGS modelling is considered to be of low importance. Indeed, with $90 \%$ TKE resolved, the effect of the SGS model can only be minor relative to the problem definition, boundary conditions and discretisation, shown to have substantial impact. Figure 12 shows the energy spectrum for a HDT field. When contrasted with Fig. 10, the effect of SGS model choice is clearly lower than that of the numerical scheme. Attention is thus focused on more critical aspects of LES for tubomachinery.

\section{Grid Resolution}

Adequate grid resolution is a critical part of predictive LES. Figure 13a shows the number of grid points required to resolve the boundary layer $N$, based on Chapmans's estimates, for a blade of aspect ratio $\approx 1$. This is plotted against Tyacke 
Table 1: Overview of resolution requirements for numerical methods (see [44]).

\begin{tabular}{lcc}
\hline Method & $\Delta x^{+} / \Delta y_{\min }^{+} / \Delta z^{+}$ & $N$ below $y^{+}=10$ \\
\hline DNS & $10-15 / 1 / 5$ & $3-5$ \\
Wall resolved LES & $50-130 / 1 / 10-30$ & $3-5$ \\
Wall modelled LES & $100-600 / 30-150 / 100-300$ & - \\
Hybrid RANS-LES & $100-600 / 1 / 100-300$ & $2-5$ \\
RANS & $1000 / 1$ & $2-5$ \\
\hline
\end{tabular}

chord-based $R e$. The $R e$ scaling of the $y^{+}<100$ and $y^{+}>100$ zones approximately represent the cost of LES and hybrid RANS-(N)LES respectively. The symbols each represent a turbomachinery LES in existing literature. Most are underresolved, the blue dash-dot-dot line indicating the linear trend. Many also attempt multi-passage or high aspect ratio blades including endwalls. Hence, a more realistic scaling is indicated by the red dash-dot trendline which highlights the incorrect $R e$ scaling. Grid resolution is hence an aspect that needs addressing. Figure 13a indicates the Re range, contrasting $N$ for different zones. At higher Re, hybrid RANS-(N)LES must be used, as using LES carries an order of magnitude increase in $N$. At low $R e$ (for example, the LPT) and flows nearly independent of $R e$ (wake-type flows), LES grid size is in the range that can currently be tackled in industry where hybrid RANS-NLES can be used for higher Reynolds number flows at similar cost. Figure 13b provides a similar plot for airframe LES. The trend is that although resolution is a little low, the gradient is nearly correct for hybrid RANS-(N)LES. This reflects the higher Re encountered in these flows and perhaps the greater attention this community pays to turbulence physics.

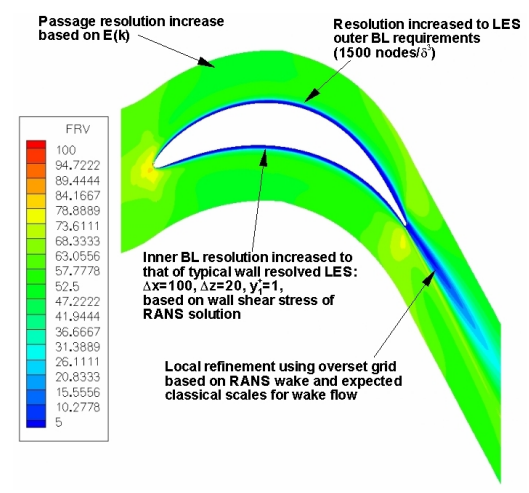

(a) RANS

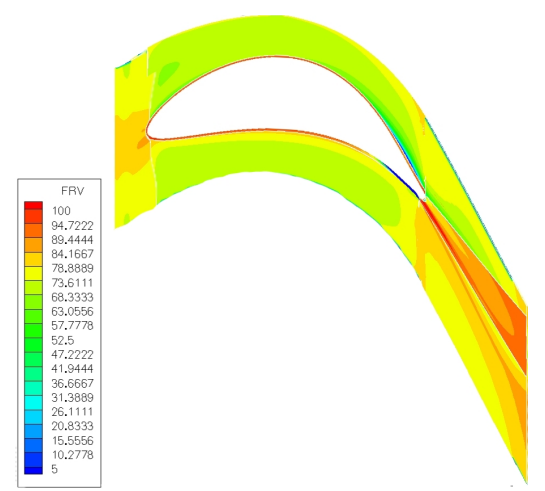

(b) LES

Fig. 14: Use of a model energy spectrum to improve grid resolution for a turbine blade.

Past validated simulations and literature provide some additional guidance on achieving adequate resolution near walls and in other zones. Table 1 provides a broad overview of resolution requirements for different numerical methods. For numerical schemes of higher order than 2, these would represent conservative estimates. For real geometries, the spacings will need realignment with the dominant flow direction. Across wakes, typically 30 nodes are required. Estimates of the wake width $\delta_{\text {wake }}$ and wall shear stress $\tau_{w}$, can be extracted from RANS. In the outer boundary layer circa 1500 points are required 


\begin{tabular}{ll}
\hline Grade & Validation level \\
\hline 1 & Integrated forces on structure - lift, drag and moments etc. \\
1.5 & Characteristic curve points of flow coefficient, sealing effectiveness etc. \\
2 & Time averaged velocity, pressure, temperature, surface adiabatic film cooling effectiveness and Nusselt number profiles, exit flow angle, total pre \\
3 & Second order statistics (r.m.s quantities) typically for turbulence \\
4 & One-point spectral analysis \\
5 & Two-point space/time-correlations \\
6 & High-order space/time correlations \\
\hline
\end{tabular}

in a $\delta^{3}$ volume [82]. A general requirement of a LES is that 80-90\% of turbulent kinetic energy $k$ is resolved [69, 70, 83]. Using a model energy spectrum [84], $E(\kappa)$ can be predicted by approximating the integral and Kolmogorov lengthscales from a RANS solution. The amount of resolved turbulent kinetic energy can then be estimated on a particular grid. Hence, using RANS and some additional heuristics, the target grid spacing (filter width) could be defined appropriately using $\Delta_{E\left(\kappa_{i}\right)}, \Delta_{\delta^{3}}, \Delta_{x_{i}}^{+}$and $\Delta_{\text {wake }}$. This method is presented in Fig. 14. Figure 14a shows an original RANS grid solution with a low percentage of resolved TKE with identified grid refinement targets indicated. Figure 14b shows a refined grid with an overset wake and boundary layer mesh, with an improved TKE resolution of around 90\%. Other resolution restrictions could be imposed for different engine zones as necessary (Refs. [85,86] also give approximate grid spacings for ribbed passages), allowing a potentially automated process. For acoustic problems, grid and temporal resolution can be estimated from the frequencies of interest, requiring 5-10 intervals per wavelength [43,87].

\section{Validation Data}

The complex flow physics targeted by LES requires more stringent validation than mean or bulk quantities. Levels of validation are provided in Tab. 2. The scale ranges from mean quantities (Grades 1-2) to higher levels involving measures of turbulence and in depth analyses.

Figure 15 indicates the percentage of literature which record grades of validation data available and the level to which LES/hybrid data is validated. The data is taken from [44], reviewing more than 200 publications. Figure 15a contrasts this for turbomachinery and $15 \mathrm{~b}$ for airframes. There is clearly a higher percentage of validation data available at higher grades in airframes than in turbomachinery. Also noteworthy in 15a is the low level of validation at grade 1 which includes integrated structural forces. The general disparity is partly due to physical restrictions in turbomachinery, though higher grades of validation data are still required to fully elucidate any shortcomings of LES. For example, SGS modelling of transitional flows and energy backscatter still remain issues [88,89]. The advantage of LES is that it will remain accurate for a wide range of flows once validated on representative cases. At low Reynolds numbers, DNS may provide validation data for specific flow physics evaluation. 


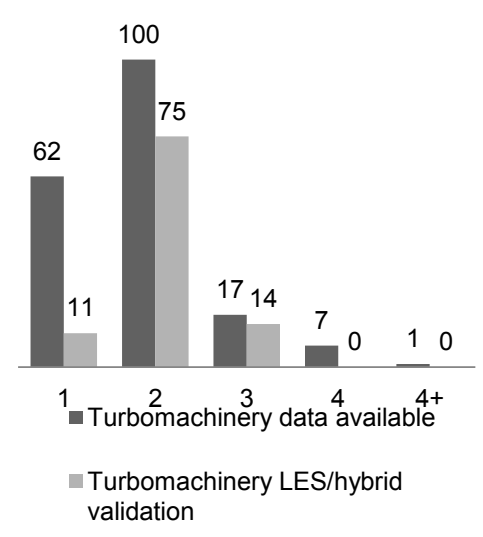

(a)

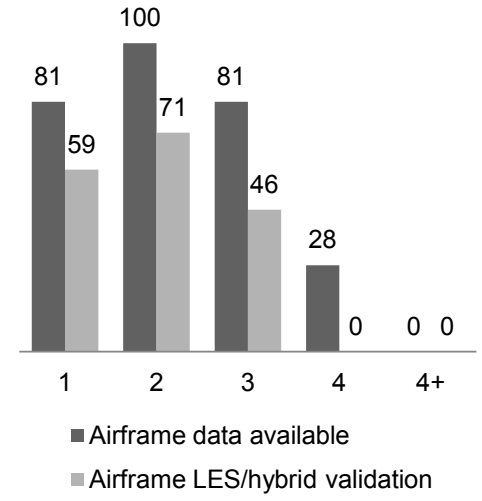

(b)

Fig. 15: Summary of validation levels contrasting turbomachinery and airframe zones (\%).

\section{Best Practices}

In some engine zones such as those containing high Re attached boundary layers, it will be hard to improve the costbenefit ratio of RANS. Here, the term benefit encompasses accuracy, quality and quantity of the data produced. At on-design conditions, compressor surface pressures are captured relatively accurately using RANS, whereas RANS accuracy degrades at off-design conditions [90]. Zones with important complex unsteady flow physics are poorly suited to RANS, requiring eddy-resolving methods to obtain sensible solutions. As an example, the accurate prediction of broadband fan noise can only be accurately predicted using eddy-resolving methods, whereas URANS/linearised solvers may be acceptable for tonal noise. As found historically with RANS turbulence modelling, there are a large number of eddy resolving methods to choose from, each with advantages and disadvantages and varying levels of turbulence resolution. Figure 16 shows an array of modelling approaches, representing a small percentage of potential solutions. An in depth discussion is inappropriate here, a recent overview can be found in [9]. The user faces a vast choice, hence best practices need to be established. Figure 17 indicates dynamic Smagorinsky (and dynamic variant) based LES is favoured in the turbomachinery field, whilst for airframes hybrid methods are preferred. Again, this may be a reflection of the $R e$ and range of complex flows found in turbomachinery.

In the authors' opinion, when using hybrid RANS-(N)LES, an interface permitting simple zonalisation based on physical quantities (i.e. not grid dependent) is required. Standard DES and a poorly trained user base could have a significant consequence. For example Jefferson-Loveday et al. [91] find the filter width used in DES inappropriate for a labyrinth seal, laminarising the flow. Previous experience with other hybrid RANS-NLES has shown promise [12,13]. A smooth interface between RANS and (N)LES zones at $60 \lesssim y^{+} \lesssim 100$ models the peak turbulence production region including near-wall streaks using URANS and (N)LES elsewhere. The work of Deck [92] is also noteworthy. Length-scales for a Zonalised-DES are given a physical basis, such that zones suited to RANS and LES are treated accordingly (see Fig. 19). For example, RANS will struggle to accurately predict the correct separation point on surfaces of low curvature, hence LES is more suitable. Such a zonalisation should be relatively straightforward for a variety of current URANS solvers. This type of approach, based on flow physics, provides a more global framework. 


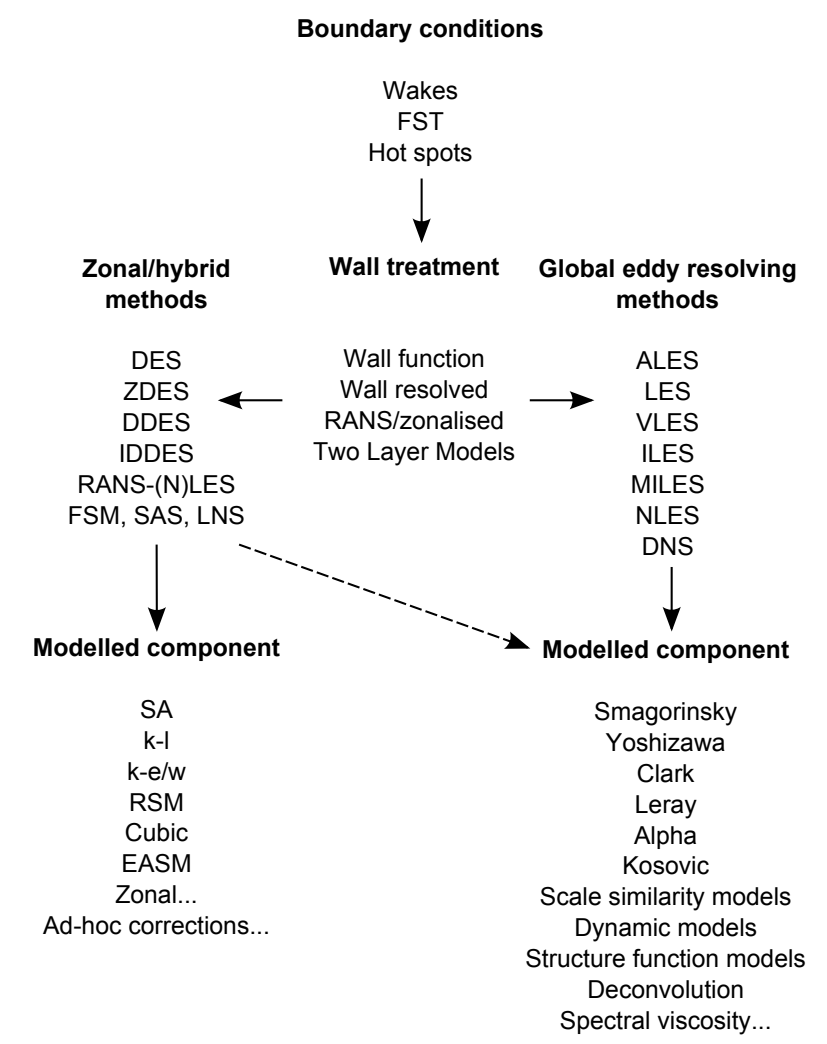

Fig. 16: Numerous choices related to eddy resolving methods.

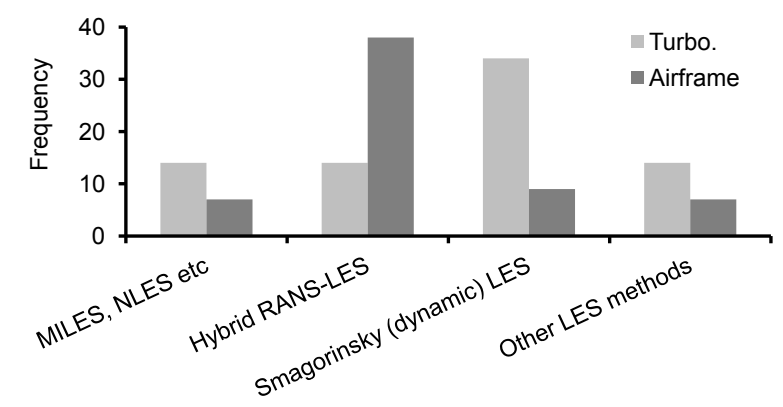

Fig. 17: Frequency of modelling methods used for turbomachinery contrasted with those for airframes.

RANS is typically poor at accurately predicting flow and heat transfer for flows involving low pressure gradient separation, recirculation, strong streamline curvature, transition and relaminarisation. LES is well suited to flows dominated by large scale turbulence. With high enough near wall resolution and suitable inflow boundary conditions LES can accurately capture transition (current computational cost generally limits this to low Reynolds numbers $O 10^{5}$ ). Generally, LES performs well where RANS begins to struggle. Conversely, the benefits of LES over RANS are diminished due to computational cost for high $R e$ attached boundary layer flows. High Re flows with a sharp geometric feature or high curvature can be tackled using hybrid RANS-LES. The effect of the RANS layer on separation is somewhat diluted by the outer LES field. Deck [92] uses geometric and boundary layer scales to identify different regions of flow. Suitable zonalisation rules could be determined for different engine zones as shown in Fig. 18. Within the broader engine zonalisation, further cost savings may also be achieved by zonalising at the componenet level. Figure 19 gives an example zonalisation for an LPT blade 


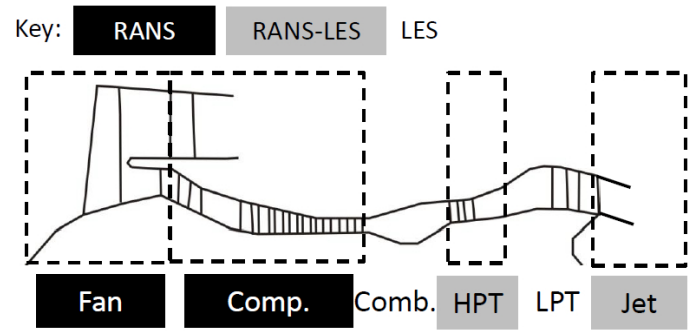

Fig. 18: Example engine-level RANS/LES zonalisation.

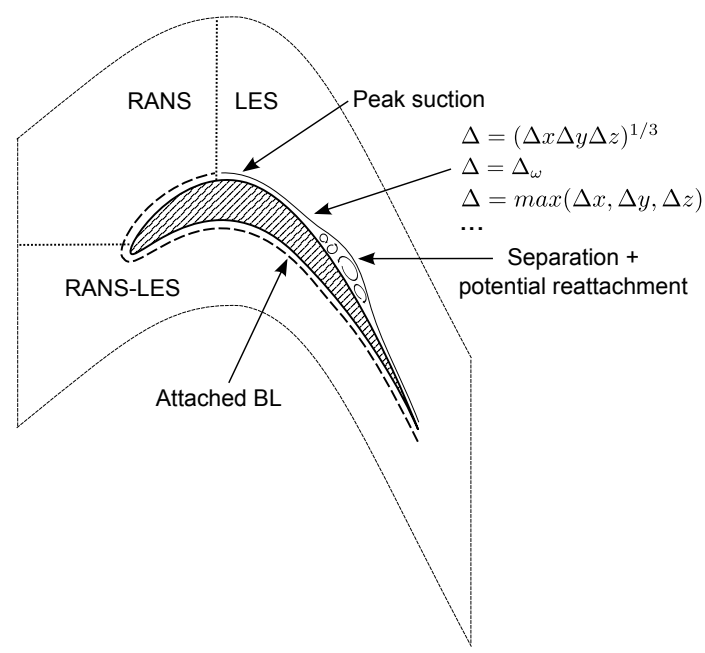

Fig. 19: Example component-level RANS/LES zonalisation based on flow features.

without incoming turbulence. The thick dashed lines show attached boundary layer (BL) regions which can be modelled using RANS or hybrid RANS-LES. On the suction surface, near the region of peak suction, which is well defined, a switch to LES is made to capture transition and separated regions. The LES filter width is then defined using appropriate scales of the flow, for example the vorticity thickness $\left(\Delta_{\omega}\right)$. It may also be noted that for LES of reacting combustor flows, explicit modelling of reactions at scales much smaller than the filter width is necessary [93] and there is a wide disparity in scales - the combustor can be physically large. The most appropriate zonalisation will depend more globally on the engine zone (limited by $R e$ for example) but also locally at the component level where specific physics is accounted for, to maximise the benefit-cost ratio. It is likely the zonalisation will change with time based on available computing resources, starting initially with LES regions for wake-type flows. The zonalisation will also define boundary condition requirements, thus defining a framework of computation that can be automated.

The initial field can have a significant impact on the time to fully develop the flow. For example, large startup vortices from a jet that need to convect out of the domain of interest. Advanced methods for initialisation and optimum convergence parameters could pay dividends. In addition, for the acoustic analysis of jets using the Ffowcs Williams-Hawkings approach [49], placement of the FWH surface is a key issue [87]. More generally, initial conditions may also affect code stability and accuracy if the flow is not fully developed. The use of coarse grids (say $1 / 8^{\text {th }}$ target resolution) to develop flowfields can provide some indication of whether the final grid is satisfactory [94], as grid resolution studies are not practical in a design Tyacke 


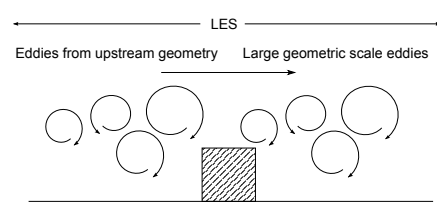

(a)

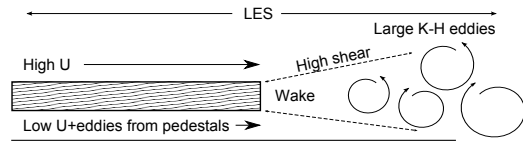

(b)

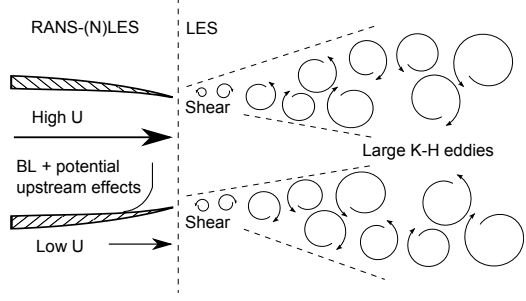

(c)

Fig. 20: Example Class A flows, (a) Ribbed ducts, (b) CBTEs, (c) predominantly free-shear flows.

context. For cascade flows, typically $2-5 t *$ (where $t *=C / U$ ) is used to develop the flow and a further $2-10 t *[95,96]$ to average statistics. For ribbed passages $t *=U_{B} / P$ with $5 t *$ for both development and averaging [85] for a periodic geometry, or the equivalent of 1 through flow to average a full serpentine passage [97]. In general, the runtime required is based on user experience, visual inspection and is problem specific. Clearly this leaves potential for errors. Short time averages to check for flow maturity and the convergence of time-mean statistics to a stationary state may be a practical manual method to monitor the flow. A more controlled method is presented by Mocket et al. [98], who introduce a relatively simple method to determine when an initial transient has passed and when enough data has been recorded that the mean is statistically converged to within given confidence intervals. This could easily be implemented as part of a solution monitoring procedure and fed back into simulation cost estimates, perhaps based on ANNs.

\section{Taxonomy}

The types of flow in different engine regions are generally well known, suggesting flows may be broadly categorised based on flow features. Inflow requirements can also be addressed in this way based on flow and upstream geometry. Restricting attention to the turbine, a potential taxonomy is shown in Fig. 20-22. The three flow classes represent flows dominated by (A), wake-type structures, (B), low-Re boundary layers and (C), high-Re boundary layers.

Class A flows are dominated by large scale structures and with little classical boundary layer content. Figure 20 shows ribbed internal cooling ducts, CBTEs and free-shear flows as examples. Generally, large scale geometrical features rapidly generate turbulence. For a ribbed internal cooling duct, [97] shows inlet turbulence has little effect on heat transfer after the second set of ribs. Strong shear or inflectional velocity profiles will also generate turbulence. Hence inlet turbulence is of low importance for these flows. Due to the large structures, grid requirements are low and pure (N)LES is suitable.

Class B flows consist of low Reynolds number boundary layer flow. Shown in Fig. 21 for an LPT, the boundary layer undergoes transition, separation and potentially reattachment. Transition is highly sensitive, hence the inclusion of incoming freestream, upstream wake turbulence and boundary layer state can be critical. The low Reynolds numbers make pure (N)LES or Quasi-DNS (QDNS) the most suitable approach.

Class C flows contain high Reynolds number boundary layers. These can be found in the HPT, or on the rotors of labyrinth and rim-seals. Figure 22 provides schematics. For the HPT, large combustor eddies will approach the leading edge, requiring compatible inflow conditions. For labyrinth and rim-seals, separation from sealing features generates large scales, 


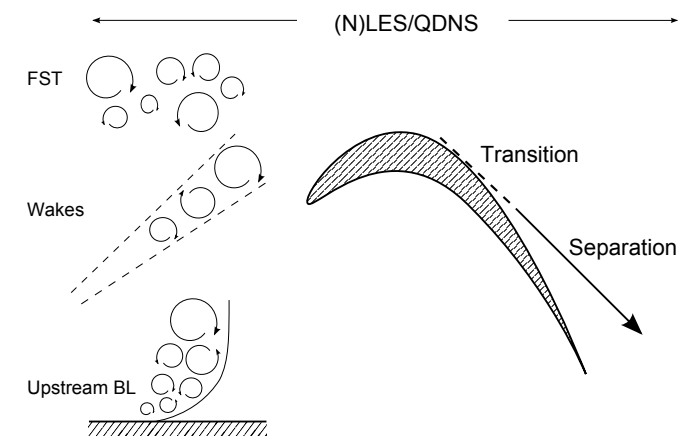

Fig. 21: Example Class B LPT flow and potential inflow requirements.

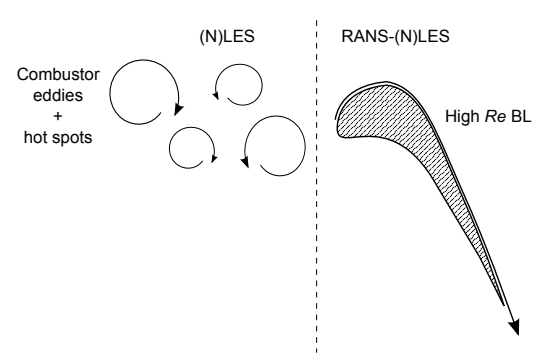

(a)

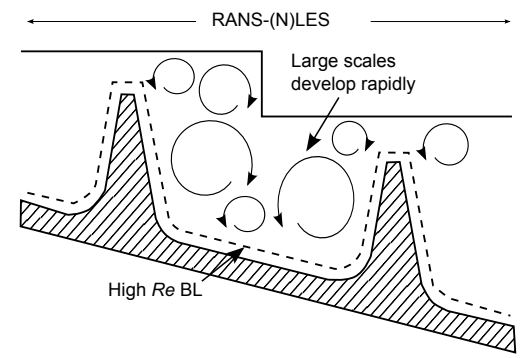

(b)

Fig. 22: Example Class C flows, (a) HPT, (b) Labyrinth/rim seals.

again lowering the importance of inflow turbulence. Although the flow type in some cases is mixed, the high $R e$ component necessitates the use of hybrid methods as in Fig. 22b. This is far from exhaustive, but the ideas could be extended for other engine zones and begins to provide an LES framework.

\section{Expert System}

In a typical industrial CFD process, the user is required to make important decisions such as which RANS model to use. This decision can dominate the solution, potentially leading to large errors. Complex analysis packages may require a year of use before the user could be regarded expert [99]. With more and more complex flows being modelled, the potential for human error increases substantially. The turbulence model choice in Fig. 16 shows each user would have to become expert in turbulence modelling to make any form of educated decision. There are hundreds of models and variants and the user still runs the risk of producing inaccurate data from poor model choice. With LES, most of the turbulence is resolved and only a small part modelled, reducing the burden of model choice.

The previous taxonomy and best practices could assist in the development of an expert system to guide design engineers $[19,20]$. This would ensure the best benefit/cost ratio at each design stage. Indeed, in the early design stages, low order models may be sufficient. However, the amount and quality of data produced by experiments or LES will be crucial in assessing designs in the later stages. Essentially the cost and benefit for using LES in different zones needs to be assessed and LES used where the benefit-cost over RANS is high. Hence quantitative benefit/cost estimates will become important. Careful application of LES will also reduce concerns over the power costs incurred, however the advances in low-power 
computing i.e. increases in FLOPS/Watt may alleviate these issues. As previously stated, FLOPS/Watt has increased by a factor of 1000 in the past ten years.

Two example expert system flow charts are presented for a ribbed internal cooling duct and LPT passage in Fig. 23a and Fig. $23 \mathrm{~b}$ respectively. The key process flows from top to bottom as indicated by the left hand side arrow. Each box (stage) has three rows, the first indicating inputs, the second, the process and the third, the output. In these the US and ES indicate input from the user or expert system respectively. Two key preliminary steps are the grid and boundary condition requirements defined by the flow classification. These can be used to estimate the cost-benefit ratio compared to alternatives such as experimental testing. If the LES is feasible, mesh generation takes place and the case is run with additional computational control and data processing. There are differences in some details reflecting the tailoring of the LES to different flow types. Figure 24 shows a trivial example of a graphical user interface for a potential expert system. The user is separated from modelling intricacies, best practices encompassed by the expert system. This will ensure consistency of LES simulations and accuracy of data.

The example provided in Fig. 23a will now be discussed. In stage 1, the user selects the engine component and CAD file using a GUI. From this, the expert system defines the flow as class A, which relies on pure LES. At stage 2a, for the ribbed passage flow, multiple intricate geometrical features exist, hence additional topology information is either provided in the CAD or extracted from the geometry, providing parameters used for mesh generation. For example, the medial axis provides a reduced order representation of the true geometry and can be used to guide mesh topology and refinement [100]. At stage $2 b$, the user defines the boundary conditions (BCs) and the expert system uses the defined flow class to infer that inlet turbulence is not required and that no additional grid points will be required to resolve inlet turbulence. Stages $2 \mathrm{a}$ and $2 \mathrm{~b}$ feed into stage 3 which estimates the total mesh size using all the available data so far. This may also include data from previous similar cases via an ANN. Stage 4 uses the current case data, and compute cluster queue status to estimate the cost and run/turnaround time. Clearly it is of no use to run an LES in one day if it takes a month to begin running. At stage 5, the user decides whether to proceed based on the current data. A summary report could be generated and recorded, feeding into HPC requirements. For the example it is assumed the user proceeds to stage 6 where the mesh is generated. At this point the expert system should have all the required information about the case to be run. For the ribbed channel, rapid mesh generation could be automatic, as the grid quality is not as critical as for the LPT. At stage 7 the case is run and standard data would be output. The user should have the opportunity to add additional output requirements whilst the case is running and any additional overheads would be presented to the user. As the solution progresses additional data could be simply requested by the user via automated post processing tools. This could perhaps be issued as a data search request. For example, "what is the $T i$ at an $x=2$ plane?". At stage 8 , the user will study the generated data and the expert system will continue to process the data, update databases and complete any other background computational tasks. The end result will enable a greater understanding of the flow, leading to improved design decisions and improvements to lower order models.

For the LPT example in Fig. 23b, the flow is defined as class B. At stage 2a, different parameters are extracted for the mesh estimates as this flow is Re dependent, hence the wetted area is required. Inlet turbulence is also important for this class of flow. Stage $2 b$ indicates turbulent inflow is required and hence the mesh requirement will be higher which feeds into stage Tyacke 


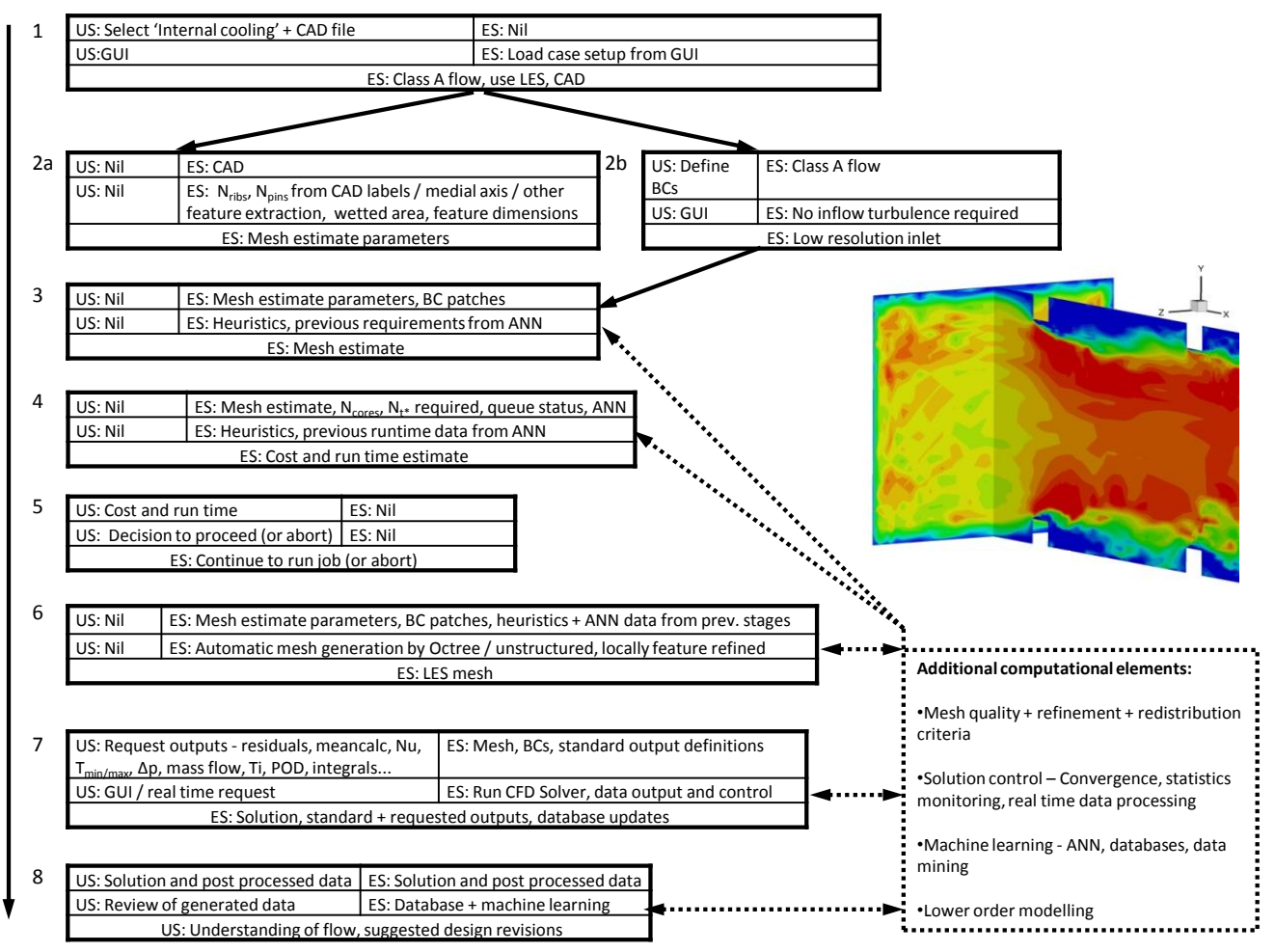

(a) Ribbed internal cooling passage.

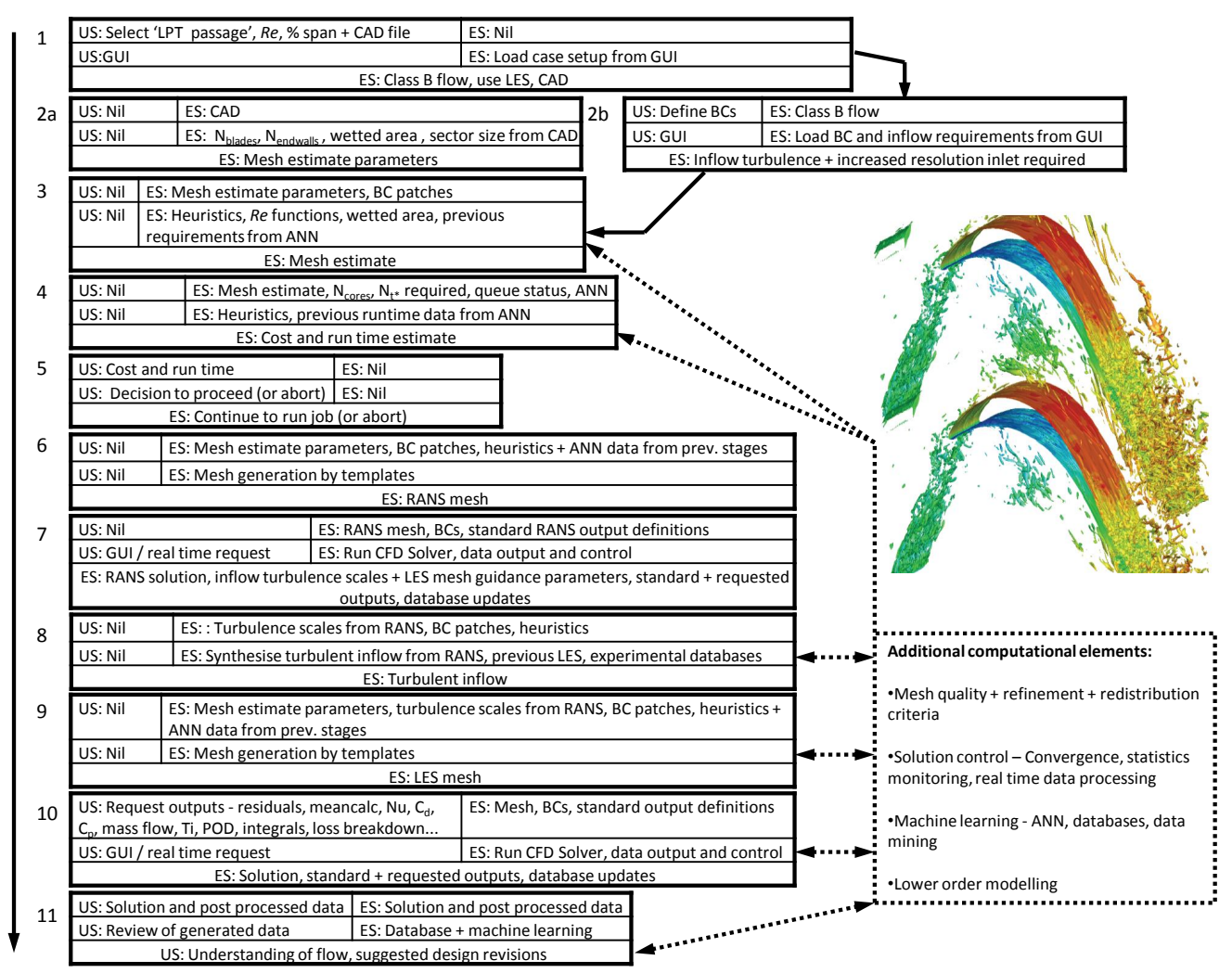

(b) LPT

Fig. 23: Examples of an expert system for internal cooling passages and an LPT blade passage. 


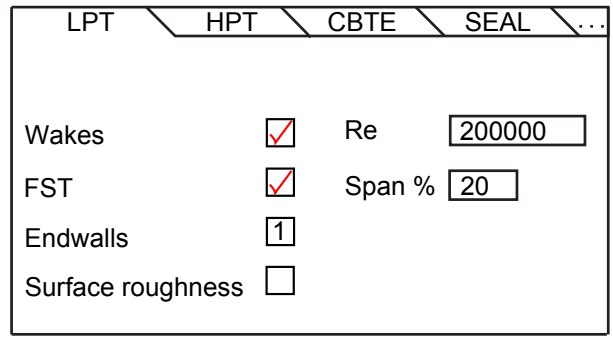

Fig. 24: Example of an expert system menu for different engine zones.

3. Here mesh estimates can be based on Re functions as shown in Fig. 13a. The process is similar until stage 6. The LPT has a relatively simple geometry that could be templated for meshing. An initial RANS mesh is generated and run in stages 6 and 7 to provide additional mesh parameters for the LES. Stage 8 generates the required inlet turbulence from the RANS and available external data. The LES mesh is generated at stage 9 by mesh templates, with adequate inlet resolution for the required inlet turbulence. Class B flows require higher quality cells than class A flows, to provide adequate resolution of transitional boundary layer zones, hence, automatic unstructured meshing currently may not be adequate. Stages 10 and 11 are effectively the same as for the ribbed passage case, except that different outputs are required for different engine zones.

Additional background computational elements are indicated by the dotted lines and surrounded by the dotted box. These would potentially oversee the simulation, for example to maintain adequate mesh quality, correlate different data sets, optimise performance and update low order models with consent. As shown in Fig. 23, the preliminary, simulation and data processing elements are broadly similar but the interdependence of different stages requires a fault tolerant, parallel, integrated tool chain. Some efforts have gone towards the development of such systems in other industries operating over different geographical regions $[101,102]$. It may also be necessary to use real-time load balancing and data management to efficiently run and extract useful data from the extremely large data sets produced. Here, algorithms adapted from large internet companies such as Google and Facebook may be useful.

Even if the simulation stage is optimised, it is still sandwiched between the pre- and post-processing stages. The preprocessing stage can be improved by speeding up robust accurate CAD [103], mesh and boundary condition generation and more efficient solution initialisation to reduce initial transients. The post processing stage can be improved by introducing real-time visualisation and analysis to monitor the flow. Statistical monitors such as MeanCalc [98] can help determine when to begin time-averaging after initial flow development. For such large data sets, user interfaces must be simplified. Data mining techniques may also be used to detect trends, flow features of interest and outliers. The entire process must become much more tightly integrated as data could be used to rapidly alter geometry or inform lower order modelling in a feed back loop. Hence, the increasing problem size and complexity will require high levels of automation and parallelism for the process to remain manageable.

\section{Interdisciplinary Interactions}

To enable the practical use of LES in turbomachinery, advances in three key areas involving significant interaction are required. As shown in Fig. 25, these are turbomachinery, applied mathematics and computer science. The turbomachinery 


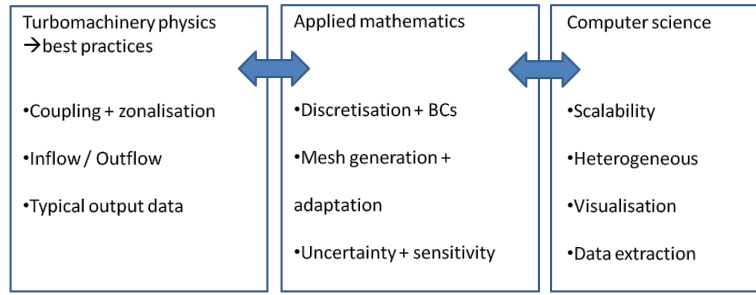

Fig. 25: Interdisciplinary interactions required.

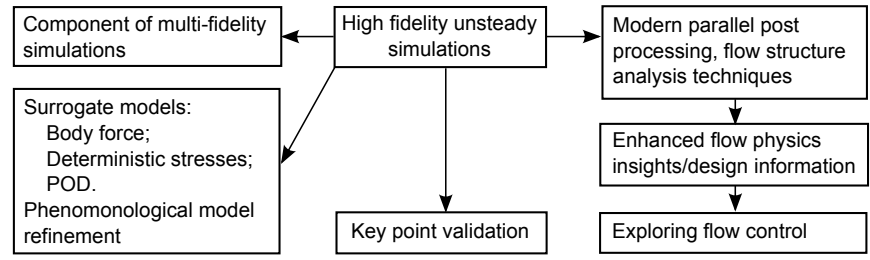

Fig. 26: Potential uses of eddy resolving simulations.

community must identify the critical flow physics to be captured by any numerical method. This will guide their determination of best practices, including coupling and zonalisation of RANS and LES zones at both the engine and component level, inflow and outflow requirements and perhaps typical data that is required. Applied mathematicians should aim to develop suitable discretisations and boundary conditions, mesh generation and adaptation algorithms and improved uncertainty and sensitivity analysis across different disciplines and fidelity levels. Computer scientists will be crucial in achieving scalability to billions of cores across multiple and constantly changing architectures. Heterogeneous computing may be considered a goal, and this may require some level of code abstraction that remains maintainable. Efficient visualisation and data extraction will also be paramount and must be possible at the same scale and pace as the simulation to prevent bottlenecks. To achieve these ambitious goals, all three disciplines will have to work closer than ever before.

\section{Current and Future Uses of Eddy Resolving Methods}

For LES to be used optimally, a narrow range of LES candidates may be identified using more traditional lower order methods. LES could be viewed as an additional unsteady modelling layer in the design process. The knowledge gained from LES creates a feedback loop, informing RANS and lower-order modelling (LOM) as shown in Fig.23.

Figure 26 provides an overview of LES uses. Experimental databases are often limited by instrumentation. LES can be used where an operating point cannot be achieved experimentally or to provide more in depth data throughout the entire flow field. LES can be used to provide in depth flow physics insights, key point validation for the improvement of lower order modelling and flow control data. Medic et al. [96] note the importance of surface roughness when performing LES of heat transfer for an HPT blade. Yang et al. [104] use DNS to evaluate turbulence budgets for real surface roughness showing an increase in skin friction of over $80 \%$ compared to a smooth wall, sand-grain only giving a $35 \%$ increase. These budgets can be used to improve RANS modelling. Tyacke et al. [105] use LES to highlight some deficiencies in RANS modelling for labyrinth seals. Jefferson-Loveday et al. [106] use LES to remedy the incorrect length-scales of the SA model in an HPT rim seal cavity, also highlighting the strong interaction of leakage with the main passage flow. LES can thus be 
used to improve RANS for particular applications, provide additional data for more empirical correlations or to fill gaps in measured data leading to improved design algorithms. LES should be used where the benefit-cost ratio is high compared to experimental testing or where RANS is unlikely to provide accurate data. For the most ideal LES flows such as CBTEs, geometry optimisation may be possible. Looking ahead to 2030, it seems possible to model 2-3 full annulus stages of a turbine/compressor or approximately $1 / 10^{\text {th }}$ full annulus of a whole engine using pure LES. Hence there are significant gains to be achieved through efficient RANS/LES zonalisation and improved algorithms, perhaps an order of magnitude in each, enabling a whole engine to be modelled with significant LES content.

\section{Conclusions}

LES is now becoming more affordable for real engineering problems. Challenges for LES in turbomachinery have historically been high Reynolds number flows. Hybrid methods can substantially reduce computational requirements, albeit with some loss in fidelity. Strong best practices are critical, considering the flow physics of each zone and making use of RANS, RANS-LES or LES where necessary. There are however zones where LES can now be applied to real geometries at near engine conditions. Low Reynolds number flows can be tackled using LES where RANS has known deficiencies, some of which may be remedied by the LES data. Wake-type flows are ideal and generally do not require complex boundary conditions. Introducing best practices and flow taxonomy and zonalisation into an expert system, provides the consistency required by industry to perform LES more routinely. To maintain growth in the use of LES methods, advances in algorithms, interfaces, parallelisation and greater systems integration of pre- and post-processing in parallel are needed. Given recent advances in computer science and performance, it now appears time to address these multiple challenges, to produce systems truly capable of exploiting available resources.

\section{References}

[1] Vogt, D. M., and Fransson, T. H., 2002. “A new turbine cascade for aeromechanical testing”. The 16th Symposium on Measuring Techniques in Transonic and Supersonic Flow in Cascades and Turbomachines, pp. 1-8.

[2] Pullan, G., Young, A. M., and Day, I. J., 2012. “Origins and Structure of Spike-Type Rotating Stall”. Proceedings of ASME Turbo Expo 2012.

[3] Tyacke, J., and Tucker, P., 2012. "LES of heat transfer in electronics". Applied Mathematical Modelling, 36(7), pp. 3112-3133.

[4] Tucker, P. G., 2011. "Computation of unsteady turbomachinery flows: Part 1 - Progress and challenges". Progress in Aerospace Sciences, 47(7), pp. 522-545.

[5] Denton, J. D., and Dawes, W. N., 1998. "Computational fluid dynamics for turbomachinery design". Proceedings of the Institution of Mechanical Engineers, Part C: Journal of Mechanical Engineering Science, 213(2), Feb., pp. 107124.

[6] Menter, F. R., 1994. "Two-Equation Eddy-Viscosity Turbulence Models for Engineering Applications”. AIAA Journal, 32(8), pp. $1598-1605$. 
[7] Launder, B. E., Reece, G. J., and Rodi, W., 1975. "Progress in the Development of a Reynolds-Stress Turbulent Closure". Journal of Fluid Mechanics, 68(3), Apr., pp. 537-566.

[8] Rumsey, C. L., Pettersson-Reif, B. A., and Gatski, T. B., 2006. “Arbitrary Steady-State Solutions with the k-epsilon Model”. AIAA Journal, 44(7), July, pp. 1586-1592.

[9] Tucker, P., 2013. “Trends in turbomachinery turbulence treatments”. Progress in Aerospace Sciences, July, pp. 1-32.

[10] Spalart, P. R., Jou, W.-H., Strelets, M., and Allmaras, S. R., 1997. “Comments on the feasibility of LES for wings, and on a hybrid RANS/LES approach”. First AFOSR International Conference on DNS/LES in Advances in DNS/LES, pp. 137-147.

[11] Spalart, P. R., 2009. "Detached-Eddy Simulation”. Annual Review of Fluid Mechanics, 41(1), Jan., pp. $181-202$.

[12] Tucker, P., Eastwood, S., Klostermeier, C., Jefferson-Loveday, R., Tyacke, J., and Liu, Y., 2010. “Hybrid LES Approach for Practical Turbomachinery Flows: Part 1 - Hierarchy and Example Simulations". ASME Conference Proceedings(44021), pp. 997-1009.

[13] Tucker, P., Eastwood, S., Klostermeier, C., Xia, H., Ray, P., Tyacke, J., and Dawes, W., 2010. "Hybrid LES Approach for Practical Turbomachinery Flows: Part 2 - Further Applications". ASME Conference Proceedings(44021), pp. 1055-1067.

[14] You, D., Wang, M., Mittal, R., and Moin, P., 2003. "Study of Rotor Tip-Clearance Flow Using Large-Eddy Simulation”. AIAA Journal, 838, pp. 1-9.

[15] Tyacke, J., Tucker, P. G., Jefferson-loveday, R., Rao Vadlamani, N., Watson, R., and Naqavi, I., 2013. “LES for Turbines: Methodologies, Cost and Future Outlooks". Proceedings of ASME Turbo Expo 2013.

[16] Chapman, D., Hans, M., and Pirtle, M. W., 1975. “Computers vs. Wind Tunnels for Aerodynamic Flow Simulations”. Astronautics and Aeronautics, 13, pp. 12-35.

[17] Qiu, X.-W., Anderson, M., and Japikse, D., 2010. “An integrated design system for turbomachinery”. Journal of Hydrodynamics, Ser. B, 22(5), Oct., pp. 358-365.

[18] Rubio, G., Valero, E., and Lanzan, S., 2012. “Computational Fluid Dynamics Expert System using Artificial Neural Networks". International Journal of Engineering and Applied Sciences, 6, pp. 40-44.

[19] Knight, B., and Petridis, M., 1992. "FLOWES: An Intelligent Computational Fluid Dynamics System”. Engng Applic. Artif. Intell., 5(1), pp. 51-58.

[20] Andrews, A. E., 1988. "Progress and challenges in the application of artificial intelligence to computational fluid dynamics". AIAA Journal, 26(1), Jan., pp. 40-46.

[21] Collado Morata, E., Gourdain, N., Duchaine, F., and Gicquel, L., 2012. "Effects of free-stream turbulence on high pressure turbine blade heat transfer predicted by structured and unstructured LES'. International Journal of Heat and Mass Transfer, 55(21-22), Oct., pp. 5754-5768.

[22] Sagaut, P., Garnier, E., Tromeur, E., Larcheveque, L., and Labourasse, E., 2004. “Turbulent Inflow Conditions for Large-Eddy Simulation of Compressible Wall-Bounded Flows”. AIAA Journal, 42(3), pp. 469-477.

[23] Jewkes, J., 2008. “An Improved Turbulent Boundary Layer Inflow Condition, Applied to the Simulation of Jets in Tyacke 
Cross-Flow". PhD thesis, University of Warwick.

[24] Keating, A., Piomelli, U., Balaras, E., and Kaltenbach, H.-J., 2004. “A priori and a posteriori tests of inflow conditions for large-eddy simulation". Physics of Fluids, 16(12), p. 4696.

[25] Tabor, G., and Baba-Ahmadi, M., 2010. "Inlet conditions for large eddy simulation: A review". Computers \& Fluids, 39(4), Apr., pp. 553-567.

[26] Lund, T. S., Wu, X., and Squires, K. D., 1998. “Generation of turbulent inflow data for spatially-developing boundary layer simulations". Journal of Computational Physics, 140(2), pp. 233-258.

[27] Schluter, J. U., Wu, X., Kim, S., Shankaran, S., Alonso, J. J., and Pitsch, H., 2005. “A Framework for Coupling Reynolds-Averaged With Large-Eddy Simulations for Gas Turbine Applications". Journal of Fluids Engineering, 127(4), pp. 806-815.

[28] $\mathrm{Wu}, \mathrm{X} ., 2010$. "Establishing the generality of three phenomena using a boundary layer with free-stream passing wakes". Journal of Fluid Mechanics, 664, Oct., pp. 193-219.

[29] Rao Vadlamani, N., 2013. "High fidelity large eddy simulation of turbines: current status and future outlook". PhD thesis, University of Cambridge.

[30] Mathey, F., Cokjlat, D., Bertoglio, J. P., and Sergent, E., 2003. "Specification of LES inlet boundary condition using vortex method". In Proceedings of the 4th International Symposium on Turbulence, Heat and Mass Transfer, Vol. 4.

[31] Kraichnan, R. H., 1970. “Diffusion by a Random Velocity Field”. Physics of Fluids, 13(1), p. 22.

[32] Labourasse, E., and Sagaut, P., 2002. "Reconstruction of Turbulent Fluctuations Using a Hybrid RANS/LES Approach”. Journal of Computational Physics, 182(1), Oct., pp. 301-336.

[33] Laraufie, R., Deck, S., and Sagaut, P., 2011. "A dynamic forcing method for unsteady turbulent inflow conditions". Journal of Computational Physics, 230(23), Sept., pp. 8647-8663.

[34] O’Mahoney, T. S. D., 2011. "Large-eddy simulation of turbine rim seals". PhD thesis, Surrey University.

[35] Klein, M., Sadiki, a., and Janicka, J., 2003. "A digital filter based generation of inflow data for spatially developing direct numerical or large eddy simulations". Journal of Computational Physics, 186(2), Apr., pp. $652-665$.

[36] Jarrin, N., Prosser, R., Uribe, J.-C., Benhamadouche, S., and Laurence, D., 2009. "Reconstruction of turbulent fluctuations for hybrid RANS/LES simulations using a Synthetic-Eddy Method”. International Journal of Heat and Fluid Flow, 30(3), June, pp. 435-442.

[37] Perret, L., Delville, J., Manceau, R., and Bonnet, J.-P., 2008. "Turbulent inflow conditions for large-eddy simulation based on low-order empirical model". Physics of Fluids, 20(7), p. 075107.

[38] Druault, P., Lardeau, S., Bonnet, J.-P., Coiffet, F., Delville, J., Lamballais, E., Largeau, J.-F., and Perret, L., 2004. “Generation of Three-Dimensional Turbulent Inlet Conditions for Large-Eddy Simulation”. AIAA Journal, 42(3), Mar., pp. 447-456.

[39] Eastwood, S., 2010. "Hybrid LES-RANS of complex geometry jets". PhD thesis, University of Cambridge.

[40] Batten, P., Goldberg, U., and Chakravarthy, S., 2004. "Interfacing Statistical Turbulence Closures with Large-Eddy Simulation”. pp. 485-492. 
[41] Xiao, F., Dianat, M., and Mcguirk, J. J., 2010. “A Recycling / Rescaling Method For LES Inlet Condition Generation”. In ERCOFTAC ETMM8 Conference, Vol. 2, no. 1992, ERCOFTAC, pp. 510-515.

[42] Wang, P. C., and Mcguirk, J. J., 2013. "Large Eddy Simulation of supersonic jet plumes from rectangular con-di nozzles”. International Journal of Heat and Fluid Flow, 43(1), pp. 62-73.

[43] Shur, M. L., Spalart, P. R., Strelets, M. K., and Travin, A. K., 2003. “Towards the prediction of noise from jet engines”. International Journal of Heat and Fluid Flow, 24, pp. 551-561.

[44] Tucker, P. G., 2013. Unsteady Computational Fluid Dynamics in Aeronautics. Springer.

[45] Hu, F. Q., 2001. “A Stable, Perfectly Matched Layer for Linearized Euler Equations in Unsplit Physical Variables”. Journal of Computational Physics, 173(2), Nov., pp. 455-480.

[46] Giles, M. B., and Carter, R. “Convergence analysis of Crank-Nicolson and Rannacher time-marching”. pp. 1-23.

[47] Pauley, L. L., Moin, P., and Reynolds, W. C., 1990. “The structure of two-dimensional separation”. J. Fluid Mech., $220(397-411)$, p. C864.

[48] Ashcroft, G., and Zhang, X., 2001. "A Computational Investigation of the Noise Radiated by Flow-Induced Cavity Oscillations". 39th AIAA Aerospace Sciences Meeting and Exhibit, pp. 1-8.

[49] Ffowcs Williams, J. E., and Hawkings, D. L., 1969. "Sound Generation by Turbulence and Surfaces in Arbitrary Motion”. Phil. Trans R. Soc. A, 264, pp. 321-342.

[50] Tam, C. K. W., 1998. “Advances in Numerical Boundary Conditions for Computational Aeroacoustics”. Journal of Computational Acoustics, 6(4), pp. 377-402.

[51] Poinsot, T. J., and K, L. S., 1992. "Boundary conditions for direct simulations of compressible viscous flows". Journal of Computational Physics, 101(1), Apr., pp. 104-129.

[52] He, L., 2013. "Fourier spectral modelling for multi-scale aero-thermal analysis". International Journal of Computational Fluid Dynamics, 27(2), pp. 118-129.

[53] Pierce, C., and Moin, P., 1998. "Method for generating equilibrium swirling inflow conditions". AIAA journal, 36(7), pp. 6-8.

[54] Schluter, J. U., Pitsch, H., and Moin, P., 2005. “Outflow Conditions for Integrated Large Eddy Simulation/ReynoldsAveraged Navier-Stokes Simulations”. AIAA Journal, 43(1), pp. 156-164.

[55] Schluter, J. U., Pitsch, H., and Moin, P., 2004. "Large Eddy Simulation Inflow Conditions for Coupling with ReynoldsAveraged Flow Solvers". AIAA, 42(3), pp. 478-484.

[56] Patil, S. S., 2011. "Large Eddy Simulations of high Reynolds number Complex Flows with Synthetic Inlet Turbulence”. PhD thesis, Virginia Polytechnic Institute and State University.

[57] Medic, G., Kalitzin, G., You, D., Herrmann, M., Ham, F., ven der Weide, E., Pitsch, H., and Alonso, J., 2006. "Integrated RANS/LES computations of turbulent flow through a turbofan jet engine". Center for Turbulence Research Annual Research Briefs, pp. 275-285.

[58] Fröhlich, J., and von Terzi, D., 2008. "Hybrid LES/RANS methods for the simulation of turbulent flows". Progress in Aerospace Sciences, 44(5), July, pp. 349-377. 
[59] He, S., Ariyaratne, C., and Vardy, a. E., 2011. "Wall shear stress in accelerating turbulent pipe flow". Journal of Fluid Mechanics, 685, Sept., pp. 440-460.

[60] Giles, M. B., 1988. “Calculation of unsteady wake/rotor interaction”. Journal of Propulsion and Power, 4(4), July, pp. 356-362.

[61] Giles, M., 1991. UNSFLO: A Numerical Method For The Calculation Of Unsteady Flow In Turbomachinery. Tech. Rep. May, GTL Report No. 205, Massachussetts Institute of Technology Gas Turbine Laboratory, Massachussetts.

[62] Connell, M., Braaten, L., Zori, R., Steed, B., Hutchinson, B., and Cox, G., 2011. "A comparison of advanced numerical techniques to model transient flow in turbomachinery blade rows". In Proceedings of the ASME Turbo Expo 2011, GT2011.

[63] Grinstein F., F., Fureby, C., and DeVore C., R., 2005. “On MILES based on flux-limiting algorithms". International Journal for Numerical Methods in Fluids, 47(10-11), pp. 1043-1051.

[64] Orzag, S. A., 1971. “Accurate solution of the Orr-Sommerfeld stability equation”. Journal of Fluid Mechanics, 50(4), pp. 689-703.

[65] Roe, P., 1981. “Approximate Riemann Solvers , Parameter Vectors and Difference Schemes”. Journal of Computational Physics, 43, pp. 357-372.

[66] Debonis, J. R., and Scott, J. N., 2002. "Study of the Error and Efficiency of Numerical Schemes for Computational Aeroacoustics”. AIAA Journal, 40(2), Feb., pp. 227-234.

[67] Jameson, A., 2007. "Formulation of Kinetic Energy Preserving Conservative Schemes for Gas Dynamics and Direct Numerical Simulation of One-Dimensional Viscous Compressible Flow in a Shock Tube Using Entropy and Kinetic Energy Preserving Schemes”. Journal of Scientific Computing, 34(2), Dec., pp. 188-208.

[68] Reynolds-Barredo, J., Newman, D., Sanchez, R., Samaddar, D., Berry, L., and Elwasif, W., 2012. "Mechanisms for the convergence of time-parallelized, parareal turbulent plasma simulations". J. Comput. Phys., 231, pp. 7851-7867.

[69] Chapman, D., 1979. “Computational aerodynamics, development and outlook”. AIAA Journal, 17(12), pp. 12931313.

[70] Choi, H., and Moin, P., 2012. “Grid-point requirements for large eddy simulation: Chapman's estimates revisited”. Physics of Fluids, 24(1), p. 11702.

[71] Peskin, C. S., 1972. "Flow patterns around heart valves: A numerical method". Journal of Computational Physics, 10, pp. 252-271.

[72] Peskin, C. S., 2002. "The immersed boundary method". Acta Numerica, 11, July, pp. 479-517.

[73] Addad, Y., Gaitonde, U., Laurence, D., and Rolfo, S., 2008. “Optimal Unstructured Meshing for Large Eddy Simulations". Quality and Reliability of Large-Eddy Simulations, 12(1), pp. 93-103.

[74] Park, M. A., 2004. “Adjoint-Based, Three-Dimensional Error Prediction and Grid Adaptation”. AIAA Journal, 42(9), pp. 1854-1862.

[75] Larsson, J., and Wang, Q., 2014. "The prospect of using LES and DES in engineering design, and the research required to get there". Phil. Trans. R. Soc. A, 372(2022), p. 20130329. 
[76] Gargallo-peir, A., Roca, X., Peraire, J., and Sarrate, J., 2013. "Defining Quality Measures for Validation and Generation of High-Order Tetrahedral Meshes". In Proceedings of the 22nd International Meshing Roundtable, Springer International Publishing, pp. 109-126.

[77] Löhner, R., and Baum, J. D., 2013. "Handling tens of thousands of cores with industrial/legacy codes: Approaches, implementation and timings". Computers \& Fluids, 85, Oct., pp. 53-62.

[78] Giles, M., and Reguly, I., 2014. “Trends in high performance computing for engineering calculations”. Phil. Trans. R. Soc. A, 372(2022), p. 20130319.

[79] Kennedy, A., and Parsons, M., 2013. “ARCHER A new UK service for academic research”. EPCC news(74), pp. 128.

[80] Kogge, P., Bergman, K., Borkar, S., Campbell, D., Carlson, W., Dally, W., Denneau, M., Franzon, P., Harrod, W., Hil, K., Hiller, J., Karp, S., Keckler, S., Klein, D., Lucas, R., Richards, M., Scarpelli, A., Scott, S., Snavely, A., Sterling, T., Williams, R. S., and Yelick, K., 2008. ExaScale Computing Study : Technology Challenges in Achieving Exascale Systems. Tech. rep., DARPA IPTO.

[81] Shacham, A., and Bergman, K., 2007. "Building ultralow-latency interconnection networks using photonic integration”. IEEE Micro, 27(4), pp. 6-20.

[82] Tucker, P., 2011. "Computation of unsteady turbomachinery flows: Part 2 - LES and hybrids". Progress in Aerospace Sciences, 47(7), Oct., pp. 546-569.

[83] Pope, S. B., 2000. Turbulent Flows. Cambridge University Press.

[84] Gamard, S., and George, W., 1999. "Reynolds Number Dependence of Energy Spectra in the Overlap Region of Isotropic Turbulence”. Flow, Turbulence and Combustion, 63, pp. 443-477.

[85] Ahn, J., Choi, H., and Lee, J. S., 2005. "Large Eddy Simulation of Flow and Heat Transfer in a Channel Roughened by Square or Semicircle Ribs". Journal of Turbomachinery, 127(2), p. 263.

[86] Viswanathan, A. K., and Tafti, D. K., 2006. "Detached eddy simulation of turbulent flow and heat transfer in a two-pass internal cooling duct”. International Journal of Heat and Fluid Flow, 27(1), pp. 1-20.

[87] Mendez, S., Shoeybi, M., Lele, S. K., and Moin, P., 2013. “On the use of the Ffowcs Williams-Hawkings equation to predict far-field jet noise from large-eddy simulations”. International Journal of Aeroacoustics, 12(1+2), pp. 1-20.

[88] Piomelli, U., Cabot, W. H., Moin, P., and Lee, S., 1991. "Subgrid-scale backscatter in turbulent and transitional flows". Physics of Fluids A: Fluid Dynamics, 3(7), p. 1766.

[89] Schlatter, P., Stolz, S., and Kleiser, L., 2004. "LES of transitional flows using the approximate deconvolution model". International Journal of Heat and Fluid Flow, 25(3), June, pp. 549-558.

[90] McMullan, W., and Page, G., 2012. "Towards Large Eddy Simulation of gas turbine compressors". Progress in Aerospace Sciences, 52, July, pp. 30-47.

[91] Jefferson-Loveday, R. J., Nagabhushana Rao, V., Tyacke, J. C., and Tucker, P. G., 2013. "High-order detached eddy simulation, zonal LES and URANS of cavity and labyrinth seal flows". International Journal for Numerical Methods in Fluids, 73(9), pp. 830-846.

Tyacke 
[92] Deck, S., 2012. “Recent improvements in the Zonal Detached Eddy Simulation (ZDES) formulation”. Theoretical and Computational Fluid Dynamics, 26(6), Oct., pp. 523-550.

[93] Pitsch, H., Desjardins, O., Balarac, G., and Ihme, M., 2008. "Large-eddy simulation of turbulent reacting flows". Progress in Aerospace Sciences, 44(6), Aug., pp. 466-478.

[94] Georgiadis, N. J., Rizzetta, D. P., and Fureby, C., 2009. "Large-Eddy Simulation : Current Capabilities , Recommended Practices, and Future Research". AIAA Journal, 48(8), pp. 1772-1784.

[95] You, D., Mittal, R., Wang, M., and Moin, P., 2004. “Computational Methodology for Large-Eddy Simulation of Tip-Clearance Flows". AIAA Journal, 42(2), pp. 271-279.

[96] Medic, G., Joo, J., Milanovic, I., and Sharma, O., 2013. "Large-Eddy Simulation for Turbine Heat Transfer". In Proceedings of ASME Turbo Expo 2013, ASME, pp. 1-9, Paper number: GT2013-95841.

[97] Sewall, E. A., and Tafti, D. K., 2008. "Large Eddy Simulation of Flow and Heat Transfer in the Developing Flow Region of a Rotating Gas Turbine Blade Internal Cooling Duct With Coriolis and Buoyancy Forces". Journal of Turbomachinery, 130(1), p. 011005.

[98] Mockett, C., Knacke, T., and Thiele, F., 2010. "Detection of initial transient and estimation of statistical error in time-resolved turbulent flow data”. In 8th International Symposium on Engineering Turbulence Modelling and Measurements - ETMM8.

[99] Jambunathan, K., Lai, E., Hartle, S., and Button, B., 1991. "Development of an intelligent front-end for a computational fluid dynamics package”. Artificial Intelligence in Engineering, 6(1), Jan., pp. 27-35.

[100] Ali, Z., and Tucker, P. G., 2013. "Multiblock Structured Mesh Generation for Turbomachinery Flows". In Proceedings of the 22nd International Meshing Roundtable, no. 20, Springer International Publishing, pp. 165-182.

[101] Smith, W., 2001. A Framework for Control and Observation in Distributed Environments. Tech. rep., NASA Ames Research Center, Moffett Field, California.

[102] Nemec, M., Aftosmis, M. J., and Pulliam, T. H., 2004. "On the Use of Parametric-CAD Systems and Cartesian Methods for Aerodynamic Design”. In Proceedings of the 3rd International Conference on Computational Fluid Dynamics, Springer-Verlag.

[103] Aftosmis, M. J., 1999. On the Use of CAD-Native Predicates and Geometry in Surface Meshing. Tech. Rep. August, NASA Ames Research Center, Moffet Field, California.

[104] Tucker, P., Goodhand, M., and Jefferson-Loveday, R., 2013. “DNS of real roughness”. In UK Turbulence Consortium Workshop, pp. 1-22.

[105] Tyacke, J., Jefferson-Loveday, R., and Tucker, P. G., 2013. "On the Application of LES to Seal Geometries”. Flow, Turbulence and Combustion, 91(4), July, pp. 827-848.

[106] Jefferson-Loveday, R., Tucker, P. G., Northall, J. D., and Rao, N., 2013. “Differential equation specification of integral turbulence length scales”. Journal of Turbomachinery, 135(3), p. 31013. 